\title{
On finite time BV blow-up for the p-system
}

\author{
Alberto Bressan ${ }^{(*)}$, Geng Chen ${ }^{(* *)}$, and Qingtian Zhang ${ }^{(* * *)}$ \\ (*) Department of Mathematics, Penn State University, \\ (**) Department of Mathematics, University of Kansas, Lawrence, \\ $(* * *)$ Department of Mathematics, University of California, Davis. \\ e-mails: bressan@math.psu.edu, gengchen@ku.edu, qzhang@math.ucdavis.edu
}

August 11, 2018

\begin{abstract}
The paper studies the possible blowup of the total variation for entropy weak solutions of the p-system, modeling isentropic gas dynamics. It is assumed that the density remains uniformly positive, while the initial data can have arbitrarily large total variation (measured in terms of Riemann invariants). Two main results are proved. (I) If the total variation blows up in finite time, then the solution must contain an infinite number of large shocks in a neighborhood of some point in the $t$-x plane. (II) Piecewise smooth approximate solutions can be constructed whose total variation blows up in finite time. For these solutions the strength of waves emerging from each interaction is exact, while rarefaction waves satisfy the natural decay estimates stemming from the assumption of genuine nonlinearity.
\end{abstract}

\section{Introduction}

For hyperbolic systems of conservation laws in one space dimension, a major remaining open problem is whether, for large BV initial data, the total variation of entropy-weak solutions remains uniformly bounded or can blow up in finite time.

In the literature, BV bounds have been established by two main approaches:

(I) Estimating the strength of new waves generated at each interaction, regardless of the order in which different wave-fronts cross each other. For small initial data, this technique was introduced by Glimm [19]. Under additional hypothesis, it can be applied also to solutions with large data. See for example [1, 26, 27, 28, 31].

(II) Relying on the decay of rarefaction waves, due to genuine nonlinearity, to provide additional cancellations. This approach first appeared in [20] and was then extended in [3, 17].

On the other hand, some particular $3 \times 3$ hyperbolic systems have been constructed in [2, 23], admitting solutions whose total variation blows up in finite time. One should remark, however, 
that these systems do not come from physical models and do not admit a strictly convex entropy.

In this paper we study the possible blowup for solutions to the p-system

$$
\left\{\begin{array}{r}
v_{t}-u_{x}=0 \\
u_{t}+p(v)_{x}=0
\end{array}\right.
$$

modeling isentropic gas dynamics in Lagrangian variables. Here $u$ is the velocity, $\rho$ is the density, $v=\rho^{-1}$ is specific volume, while $p=p(v)$ is the pressure.

In [5, 6], for a general class of pressure functions $p(\cdot)$, the authors constructed piecewise constant approximate solutions whose total variation grows without bound. For these front tracking approximations, the strength of wave fronts emerging at each interaction is the same as in the exact solution, while the only source of error is in the wave speeds. These examples confirm the analysis in [16], and show that uniform BV bounds cannot be established relying only on an accurate estimate of wave strengths across interations.

The next question, which we investigate in the present paper, is whether BV bounds for the p-system can be established by taking into account also the decay of rarefaction waves, stemming from the assumption $p^{\prime \prime}(v)>0$. We recall that Oleinik-type estimates on the decay of positive waves for genuinely nonlinear $n \times n$ hyperbolic systems were proved in [4, 8, 11. The analysis in the last section of [5] shows that, if this decay of rarefaction waves were taken into account, then the interaction patterns considered in [5, 6] would no longer yield a large amplification of total variation. It is thus natural to ask:

(Q) Consider a piecewise smooth approximate solution of (1.1) with large BV initial data. Assume that

- at each interaction, the strengths of outgoing waves are the same as in an exact solution,

- rarefaction waves satisfy decay estimates as in [4, 8, 11], due to genuine nonlinearity,

- the density remains uniformly positive.

Can the total variation still blow up in finite time?

An example will be constructed, showing that finite time BV blowup for such approximate solutions is indeed possible.

Although our solutions are not exact, because some errors occur in the wave speeds, they possess all the qualitative properties known for exact solutions. The present analysis thus provides some indication that finite time blowup of the total variation might be possible, for the p-system.

Our second main result yields a necessary condition for blowup. Namely, we prove that if the total variation blows up in finite time, then the solution must contain an infinite number of large shocks, in a neighborhood of some point in the $t-x$ plane.

This result should be compared with earlier literature, proving BV stability for various classes of initial data

$$
u(0, x)=\bar{u}(x), \quad v(0, x)=\bar{v}(x) .
$$


- If $\bar{u}, \bar{v}$ have sufficiently small total oscillation, in the sense that all the initial values $(\bar{u}(x), \bar{v}(x))$ are contained in a disc with sufficiently small radius, in the $v$ - $u$ plane, then the solution of (1.1) exists globally in time. Moreover, bounds on the total variation can be provided, uniformly in time [20].

- If $\bar{u}, \bar{v}$ are a sufficiently small BV perturbation of some (possibly large) Riemann data, then again the solution exists globally in time and its total variation remains uniformly bounded [7, 24].

Building upon these ideas, our present analysis shows that, for a solution containing only finitely many large shocks, the total variation remains bounded. Indeed, the blow-up of the BV norm in finite time requires the presence of infinitely many large shocks in a bounded region of the $t-x$ plane.

The remainder of the paper is organized as follows. To keep the exposition self-contained, in Section 2 we review some well known results on the interaction of elementary waves for the psystem. Section 3 develops some estimates related to the decay of rarefaction waves, valid also for solutions with large oscillation. In Section 4 we construct a piecewise constant approximate solution with a periodic interaction pattern, and where all rarefaction waves decay at the rate $1 / t$. By a suitable modification of this basic pattern, in Section 5, we construct a piecewise smooth approximate solution whose BV norm blows up in finite time.

Section 6 contains the statement of our main theorem, providing a necessary condition for finite time blowup. Details of the proof are then worked out in Sections 7 and 8.

\section{Elementary wave interactions for the p-system}

Throughout this paper we consider the p-system (1.1) with $\gamma$-law pressure

$$
p(v)=A v^{-\gamma}=A \rho^{\gamma},
$$

for some constants $\gamma>1$ and $A>0$. For this system one can define the Riemann invariants $w_{1}$ and $w_{2}$ by setting

$$
w_{1} \doteq u-h \quad w_{2} \doteq u+h
$$

where

$$
h \doteq B v^{(1-\gamma) / 2}=B \rho^{(\gamma-1) / 2}, \quad B \doteq \frac{2}{\gamma-1} \sqrt{A \gamma}
$$

For future use, we record the identities

$$
v=\left(\frac{h}{B}\right)^{2 /(1-\gamma)}=\left(\frac{(\gamma-1)\left(w_{2}-w_{1}\right)}{4 \sqrt{A \gamma}}\right)^{2 /(1-\gamma)} .
$$

For any smooth solution, these Riemann invariants remain constant along forward and backward characteristics, respectively. Namely

$$
w_{1, t}-c w_{1, x}=0, \quad w_{2, t}+c w_{2, x}=0,
$$

where the (Lagrangian) wave speed $c$ is

$$
c=\sqrt{-p^{\prime}(v)}=\sqrt{A / \gamma} \cdot v^{-(\gamma+1) / 2} .
$$


In the following, it will be convenient to express the wave speed in terms of the Riemann coordinates $w_{1}, w_{2}$ in (2.2). Introducing the function

$$
c(s)=\sqrt{A / \gamma} \cdot\left(\frac{(\gamma-1) s}{4 \sqrt{A \gamma}}\right)^{(\gamma+1) /(\gamma-1)},
$$

by (2.4) and (2.6) the wave speed can be written as

$$
\sqrt{-p^{\prime}(v)}=c\left(w_{2}-w_{1}\right)
$$

Example. In the special case where $p=\rho^{3} / 3$, one has the simple relation $h=\rho$. By (2.2) and (2.6), the Riemann invariants and the wave speed are given by

$$
w_{1}=u-\rho, \quad w_{2}=u+\rho, \quad c=\rho^{2} .
$$

\subsection{Elementary waves.}

A solution to the p-system contains three types of waves: rarefactions, compressions, and shock waves. In terms of the variable $h$ at (2.3), left and right states will be denoted by

$$
\left(u_{-}, h_{-}\right) \quad \text { and } \quad\left(u_{+}, h_{+}\right) \text {, }
$$

respectively.

Recalling (2.2), the signed wave strength will always be measured in terms of Riemann invariants:

$$
w_{1,+}-w_{1-} \text { for a } 1 \text {-wave, } \quad w_{2,+}-w_{2,-} \text { for a } 2 \text {-wave. }
$$

We now recall the construction of basic wave curves. See [4, 29] for details.

1. The rarefaction and compression waves satisfy the following equations:

- For a 1-wave (backward moving front),

$$
u_{+}-u_{-}=h_{-}-h_{+}
$$

where $h_{+}>h_{-}$for a 1 -compression wave and $h_{+}<h_{-}$for 1 -rarefaction wave.

- For a 2-wave (forward moving front),

$$
u_{+}-u_{-}=h_{+}-h_{-}
$$

where $h_{+}<h_{-}$for a 2 -compression wave and $h_{+}>h_{-}$for a 2-rarefaction wave. 
2. A shock wave with left state $\left(u_{-}, \rho_{-}\right)$and right state $\left(u_{+}, \rho_{+}\right)$, traveling with speed $\lambda$, satisfies the Rankine-Hugoniot equations

$$
\left\{\begin{aligned}
\lambda\left(\frac{1}{\rho_{+}}-\frac{1}{\rho_{-}}\right) & =u_{-}-u_{+}, \\
\lambda\left(u_{+}-u_{-}\right) & =p\left(v_{+}\right)-p\left(v_{-}\right)=A \rho_{+}^{\gamma}-A \rho_{-}^{\gamma} .
\end{aligned}\right.
$$

The Lax admissibility condition here yields $u_{+}<u_{-}$for both 1 -waves and 2-waves. Hence

$$
u_{+}-u_{-}=-\sqrt{\left(\frac{1}{\rho_{+}}-\frac{1}{\rho_{-}}\right)\left(A \rho_{-}^{\gamma}-A \rho_{+}^{\gamma}\right)},
$$

and

$$
\lambda= \pm \sqrt{A} \sqrt{\frac{\rho_{-}^{\gamma}-\rho_{+}^{\gamma}}{\frac{1}{\rho_{+}}-\frac{1}{\rho_{-}}}} .
$$

For a 1-shock one has $\rho_{+}>\rho_{-}$, while for a 2-shock one has $\rho_{+}<\rho_{-}$.

The following observation will be useful. Setting

$$
s=u_{-}-u_{+}, \quad \theta=\frac{\rho_{+}}{\rho_{-}}=\left(\frac{h_{+}}{h_{-}}\right)^{\frac{2}{\gamma-1}},
$$

from (2.10) it follows that, for any shock wave,

$$
s=\frac{h_{-}}{B} \sqrt{\frac{A(1-\theta)\left(1-\theta^{\gamma}\right)}{\theta}},
$$

where $B$ is the constant at (2.3) .

\subsection{Small wave interactions}

Next, we review some well known results on wave interactions, for future use. Note that, when a wave-front crosses a shock or a compression of the opposite family, the density $\rho$ (and hence $h$ as well) increases. On the other hand, the density along a wave-front decreases when it crosses a rarefaction of the opposite family.

For any pairwise interaction between two small (shock or rarefaction) waves, one has the following estimates (see [4, 20, 29]).

Proposition 2.1 Call $\sigma^{\prime}, \sigma^{\prime \prime}$ the strengths of two interacting wave-fronts, and let $\sigma_{1}, \sigma_{2}$ be the strengths of the outgoing waves of the first and second family, in the solution of the Riemann problem. Then there exists a constant $C_{0}$ (uniformly valid as the state of the system ranges over a bounded set in the $\rho$-u plane, with $\rho$ bounded away from zero) such that

- If $\sigma^{\prime}$ is a 1-wave and $\sigma^{\prime \prime}$ is a 2-wave, then

$$
\left|\sigma_{1}-\sigma^{\prime}\right|+\left|\sigma_{2}-\sigma^{\prime \prime}\right| \leq C_{0}\left|\sigma^{\prime} \sigma^{\prime \prime}\right|\left(\left|\sigma^{\prime}\right|+\left|\sigma^{\prime \prime}\right|\right) .
$$


- If both $\sigma^{\prime}$ and $\sigma^{\prime \prime}$ belong to the first family, then

$$
\left|\sigma_{1}-\left(\sigma^{\prime}+\sigma^{\prime \prime}\right)\right|+\left|\sigma_{2}\right| \leq C_{0}\left|\sigma^{\prime} \sigma^{\prime \prime}\right|\left(\left|\sigma^{\prime}\right|+\left|\sigma^{\prime \prime}\right|\right) .
$$

- If both $\sigma^{\prime}$ and $\sigma^{\prime \prime}$ belong to the second family, then

$$
\left|\sigma_{1}\right|+\left|\sigma_{2}-\left(\sigma^{\prime}+\sigma^{\prime \prime}\right)\right| \leq C_{0}\left|\sigma^{\prime} \sigma^{\prime \prime}\right|\left(\left|\sigma^{\prime}\right|+\left|\sigma^{\prime \prime}\right|\right) .
$$

\subsection{A rarefaction or compression wave crosses a large shock.}
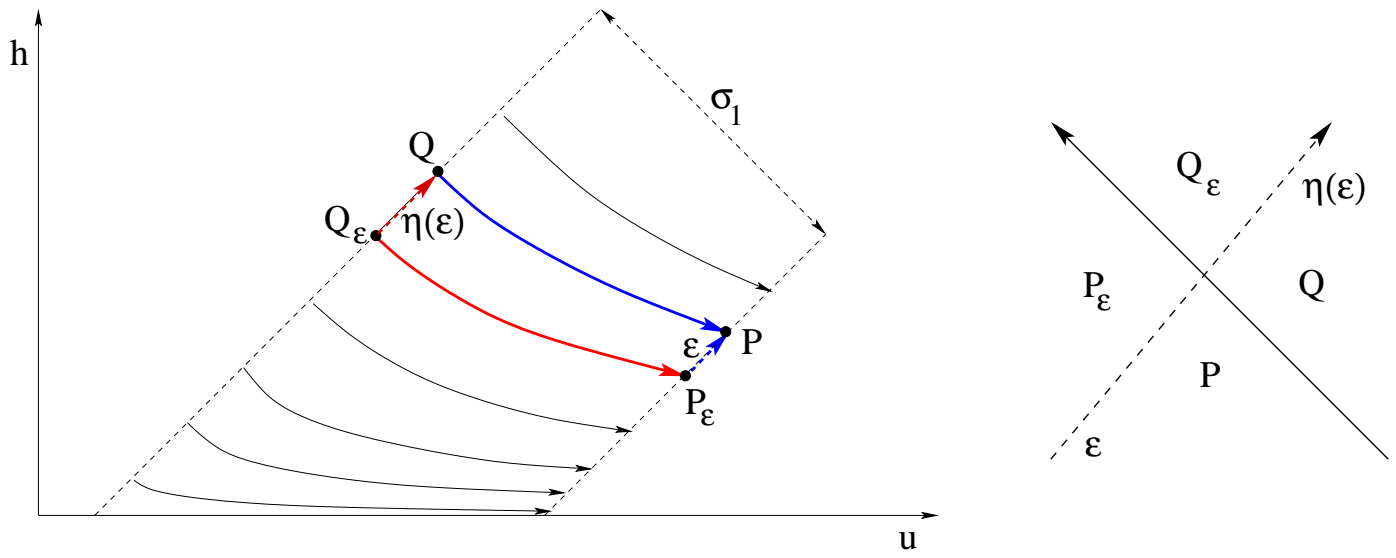

Figure 1: A small 2-rarefaction crosses a 1-shock. In this configuration, the strength of the shock does not change, while the strength of the rarefaction increases from $\varepsilon$ to some value $\eta(\varepsilon)>\varepsilon$.

To fix the ideas, consider a large 1-shock which crosses a small 2-wave (compression or rarefaction) of size $\sigma_{2}=\varepsilon$. We seek an estimate on the size of the outgoing waves, up to leading order. As shown in Fig. 1, let

$$
P=\left(u_{-}, h_{-}\right), \quad Q=\left(u_{+}, h_{+}\right)
$$

be the left and right states across the large 1-shock before the interaction, and let

$$
P_{\varepsilon}=\left(u_{-}-\varepsilon, h_{-}-\varepsilon\right), \quad Q_{\varepsilon}=\left(u_{+}-\eta(\varepsilon), h_{+}-\eta(\varepsilon)\right)
$$

be the left and right states across the 1-shock after the interaction. Set

$$
s(\varepsilon) \doteq\left(u_{-}-\varepsilon\right)-\left(u_{+}-\eta(\varepsilon)\right), \quad \theta(\varepsilon) \doteq\left(\frac{h_{+}-\eta(\varepsilon)}{h_{-}-\varepsilon}\right)^{\frac{2}{\gamma-1}} .
$$

By (2.13), replacing $P$ with $P_{\varepsilon}$ we can write

$$
s(\varepsilon)=\frac{h_{-}-\varepsilon}{B} \sqrt{\psi(\theta(\varepsilon))}, \quad \text { with } \quad \psi(\theta) \doteq \frac{A(\theta-1)\left(\theta^{\gamma}-1\right)}{\theta} .
$$

Hence

$$
\eta(\varepsilon)-\varepsilon=s(\varepsilon)-\left(u_{-}-u_{+}\right)=\frac{h_{-}-\varepsilon}{B} \sqrt{\psi(\theta(\varepsilon))}-\left(u_{-}-u_{+}\right) .
$$


Differentiating w.r.t. $\varepsilon$ we obtain

$$
\begin{aligned}
& \eta^{\prime}(\varepsilon)-1=-\frac{1}{B} \sqrt{\psi(\theta(\varepsilon))}+\frac{h_{-}-\varepsilon}{2 B \sqrt{\psi(\theta(\varepsilon))}} \cdot \psi^{\prime}(\theta(\varepsilon)) \theta^{\prime}(\varepsilon), \\
& \theta^{\prime}(\varepsilon)=\frac{2}{\gamma-1}[\theta(\varepsilon)]^{(3-\gamma) / 2} \cdot \frac{-\eta^{\prime}(\varepsilon)\left(h_{-}-\varepsilon\right)+\left(h_{+}-\eta(\varepsilon)\right)}{\left(h_{-}-\varepsilon\right)^{2}} .
\end{aligned}
$$

By (2.17), at $\varepsilon=0$ the above expression reduces to

$$
\theta^{\prime}=\frac{2}{\gamma-1} \theta^{(3-\gamma) / 2} \cdot \frac{-\eta^{\prime}+\theta^{(\gamma-1) / 2}}{h_{-}}
$$

Using (2.21) to compute the right hand side of (2.20), when $\varepsilon=0$ we find

$$
\eta^{\prime}-1=-\frac{1}{B} \sqrt{\psi(\theta)}+\frac{1}{2 B \sqrt{\psi(\theta)}} \cdot \psi^{\prime}(\theta) \cdot \frac{2}{\gamma-1} \theta^{(3-\gamma) / 2} \cdot\left(-\eta^{\prime}+\theta^{(\gamma-1) / 2}\right)
$$

Solving for $\eta^{\prime}$, we finally obtain

$$
\eta^{\prime}=\frac{2 B \sqrt{\psi}-2 \psi+\frac{2}{\gamma-1} \theta \psi^{\prime}}{2 B \sqrt{\psi}+\frac{2}{\gamma-1} \theta^{(3-\gamma) / 2} \psi^{\prime}} \doteq a(\theta)
$$

We observe that $\eta^{\prime}$ is the factor by which an infinitesimal 2-wave (either a compression or a rarefaction) is amplified when it crosses the 1-shock. According to (2.24), this ratio depends only on $\theta$. In particular, as $\theta$ remains bounded, the above amplification coefficient is a bounded number. To compute the amplification of an arbitrary rarefaction or compression wave which crosses a large shock of the opposite family, we can simply integrate (2.24) and obtain

$$
\eta(\bar{\varepsilon})=\int_{0}^{\bar{\varepsilon}} a(\theta(\varepsilon)) d \varepsilon
$$

By a direct calculation we now prove $a(\theta)>1$. In other words, as a compression or rarefaction wave crosses a shock of the opposite family, its strength always increases. In view of (2.24), this will be a consequence of the two inequalities

$$
\begin{gathered}
\left(2 B \sqrt{\psi}-2 \psi+\frac{2}{\gamma-1} \theta \psi^{\prime}\right)-\left(2 B \sqrt{\psi}+\frac{2}{\gamma-1} \theta^{\frac{3-\gamma}{2}} \psi^{\prime}\right)>0 \\
2 B \sqrt{\psi}+\frac{2}{\gamma-1} \theta^{\frac{3-\gamma}{2}} \psi^{\prime}>0 .
\end{gathered}
$$

We begin by observing that $\theta=h_{+} / h_{-}>1$, and hence

$$
\psi(\theta) \doteq \frac{A(\theta-1)\left(\theta^{\gamma}-1\right)}{\theta}>0, \quad \psi^{\prime}(\theta)=A \theta^{-2}\left[-1+(1-\gamma) \theta^{\gamma}+\gamma \theta^{\gamma+1}\right]>0,
$$


proving (2.27). Moreover, one has

$$
\begin{aligned}
& \left(2 B \sqrt{\psi}-2 \psi+\frac{2}{\gamma-1} \theta \psi^{\prime}\right)-\left(2 B \sqrt{\psi}+\frac{2}{\gamma-1} \theta^{\frac{3-\gamma}{2}} \psi^{\prime}\right) \\
& =-2 \psi+\frac{2}{\gamma-1} \theta \psi^{\prime}-\frac{2}{\gamma-1} \theta^{\frac{3-\gamma}{2}} \psi^{\prime} \\
& =-2 A\left(\theta^{-1}+\theta^{\gamma}-\theta^{\gamma-1}-1\right)+\frac{2}{\gamma-1}\left(-A \theta^{-1}+A(1-\gamma) \theta^{\gamma-1}+A \gamma \theta^{\gamma}\right)\left(1-\theta^{\frac{1-\gamma}{2}}\right) \\
& =\frac{2 A}{\gamma-1}\left(1-\theta^{-\frac{\gamma+1}{2}}\right)\left[-\gamma \theta^{\frac{\gamma-1}{2}}(\theta-1)+\left(\theta^{\gamma}-1\right)\right]>0 .
\end{aligned}
$$

Indeed, to see that the last factor on the right hand side of (2.29) is positive for $\theta>1$, we set

$$
f(\theta)=-\gamma \theta^{\frac{\gamma-1}{2}}(\theta-1)+\theta^{\gamma}-1=-\gamma \theta^{\frac{\gamma+1}{2}}+\gamma \theta^{\frac{\gamma-1}{2}}+\theta^{\gamma}-1 .
$$

Then

$$
f(1)=0, \quad \quad f^{\prime}(\theta)=\gamma \theta^{\frac{\gamma-3}{2}}\left[-\frac{\gamma+1}{2} \theta+\frac{\gamma-1}{2}+\theta^{\frac{\gamma+1}{2}}\right]=\gamma \theta^{\frac{\gamma-3}{2}} \cdot g(\theta),
$$

where

$$
\begin{gathered}
g(\theta)=-\frac{\gamma+1}{2} \theta+\frac{\gamma-1}{2}+\theta^{\frac{\gamma+1}{2}}, \\
g(1)=0, \quad g^{\prime}(\theta)=-\frac{\gamma+1}{2}+\frac{\gamma+1}{2} \theta^{\frac{\gamma-1}{2}}>0 .
\end{gathered}
$$

For $\theta>1$ we thus have $g(\theta)>0$, hence $f^{\prime}(\theta)>0$ and $f(\theta)>0$. This completes the proof that $a(\theta)>1$ for $\theta>1$.

With reference to Figure 1, the inequality $|\eta(\varepsilon)|>|\varepsilon|$ implies that the $h$-components of the states $P, Q, P_{\varepsilon}, Q_{\varepsilon}$ satisfy

$$
\left|h_{Q}-h_{Q^{\prime}}\right|>\left|h_{P}-h_{P^{\prime}}\right|
$$

\subsection{A small shock crosses a large shock}
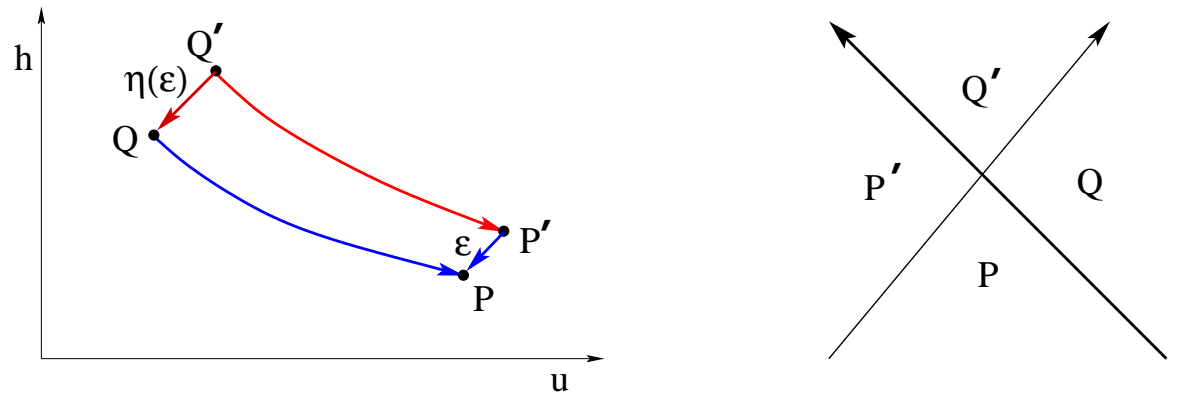

Figure 2: A small 2-shock crosses a large 1-shock. 
Let $\varepsilon<0$ be the signed strength of the small shock. Since shock and rarefaction curves have a second-order tangency [4, 29], with the notation used in Fig. 2] we have

$$
\begin{aligned}
& P=\left(u_{-}, h_{-}\right), \quad Q=\left(u_{+}, h_{+}\right), \\
& P^{\prime}=\left(u_{-}-\varepsilon+o\left(\varepsilon^{2}\right), h_{-}-\varepsilon+o\left(\varepsilon^{2}\right)\right), \quad Q^{\prime}=\left(u_{+}-\eta(\varepsilon)+o\left(\varepsilon^{2}\right), h_{+}-\eta(\varepsilon)+o\left(\varepsilon^{2}\right)\right) .
\end{aligned}
$$

Here and in the sequel, the Landau notation $o\left(\varepsilon^{2}\right)$ denotes an infinitesimal of higher order w.r.t. $\varepsilon^{2}$. Computing the derivative $\eta^{\prime}$ at $\varepsilon=0$, we thus recover exactly the same expression as in (2.23).

Because of the second order tangency condition, the change in the strength of the big shock will also be of order $o\left(\varepsilon^{2}\right)$.

We conclude with an estimate which will be used later.

Lemma 2.1 Fix $0<a<b$ and consider the Riemann problem determined by the interaction of a large 1-shock with another wave front of strength $|\sigma|$. Assume that the left, middle, and right states remain in the region where $a \leq h(v) \leq b$. Call $\left|\sigma^{\prime}\right|$ the strength of the outgoing 2-wave generated by the interaction. Then there exists a constant $C_{\gamma}$ depending only on $a, b$ such that:

(i) If the second wave impinges on the 1-shock from the left, then strength of the outgoing 2-wave satisfies

$$
\left|\sigma^{\prime}\right| \leq C_{\gamma}|\sigma|
$$

(ii) If the second wave is a 1-shock, or a small 1-compression or 1-rarefaction, impinging on the 1-shock from the right, then strength of the outgoing 2-wave satisfies

$$
\left|\sigma^{\prime}\right| \leq|\sigma|
$$

Proof. Part (i) is an immediate consequence of Proposition 2.1.

Toward a proof of (ii), we first recall a basic property of shock curves for the p-system. Fix a left state $\left(u_{-}, h_{-}\right)$, and consider the curve of all points $\left(u_{+}, h_{+}\right)$which can be connected to $\left(u_{-}, h_{-}\right)$by a 1 -shock. Writing $h_{=}=h_{+}\left(u_{+}\right)$, the slope of this curve satisfies

$$
-1 \leq \frac{d h_{+}}{d u_{+}}<0 .
$$

Indeed, this inequality is established within the proof of Lemma 3 in [28. It is also found in Section 3 of [16].

Next, assume that the left and right states across the large 1-shock are

$$
P=\left(u_{P}, h_{P}\right), \quad Q=\left(u_{Q}, h_{Q}\right),
$$

Two cases will be considered.

CASE 1: The impinging 1-wave is a shock, connecting the states $Q, Q^{\prime}$. As shown in Fig. 3 , the outgoing 2-rarefaction connects the states $W, Q^{\prime}$, where $W=\left(u_{W}, h_{W}\right)$ is the unique state along the 1-shock curve through $P$ such that

$$
u_{Q^{\prime}}-h_{Q^{\prime}}=u_{W}-h_{W} .
$$




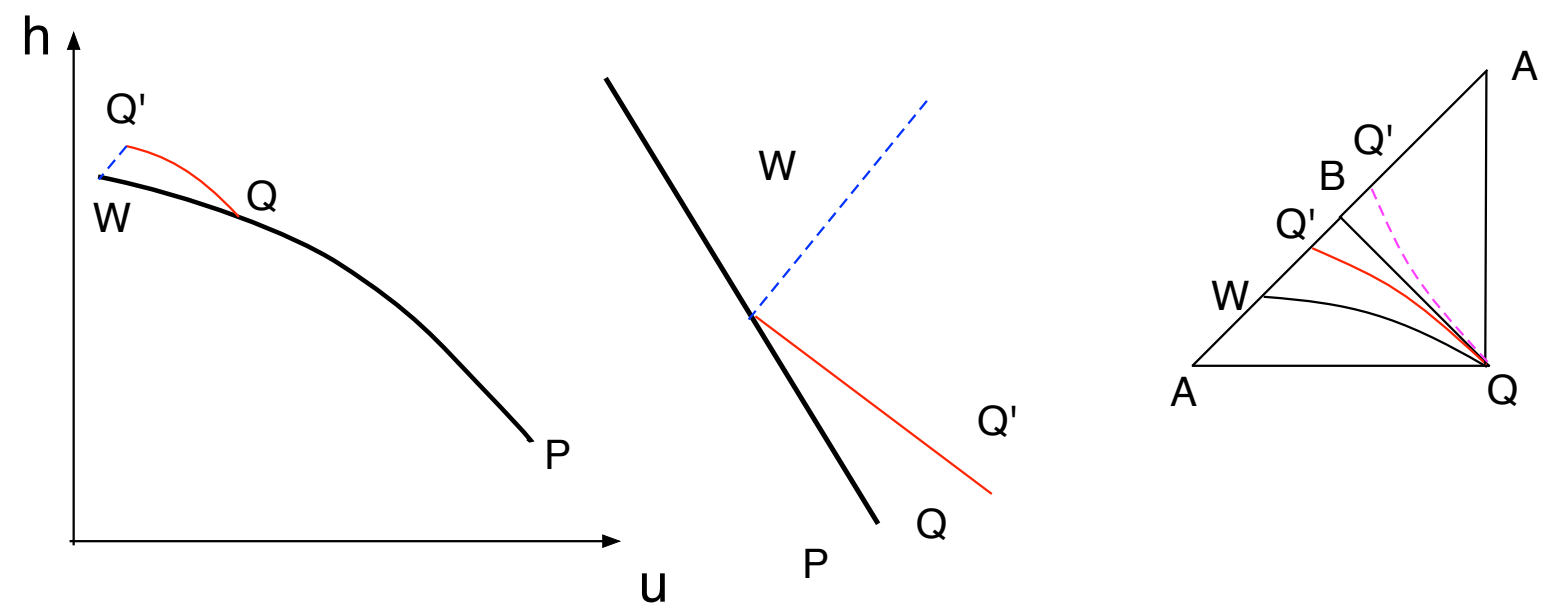

Figure 3: A small 1-shock impinges a large 1-shock.

In this case, call $A$ and $B$ the points in the $u$ - $h$ plane such that

$$
\begin{gathered}
u_{Q^{\prime}}-h_{Q^{\prime}}=u_{A}-h_{A}=u_{B}-h_{B}, \\
h_{A}=h_{Q}, \quad u_{B}+h_{B}=u_{Q}+h_{Q} .
\end{gathered}
$$

We then have

$$
\begin{aligned}
& \text { [strength of the outgoing 2-rarefaction] }=\left|\left(u_{Q^{\prime}}+h_{Q^{\prime}}\right)-\left(u_{W}+h_{W}\right)\right| \\
& \quad \leq\left(u_{B}+h_{B}\right)-\left(u_{A}+h_{A}\right)=\left(u_{Q}-h_{Q}\right)-\left(u_{B}-h_{B}\right) \\
& \quad=\left(u_{Q}-h_{Q}\right)-\left(u_{Q^{\prime}}-h_{Q^{\prime}}\right)=\text { [strength of the incoming 1-shock]. }
\end{aligned}
$$

CASE 2: The incoming 1-wave is a compression. In this case we have $Q^{\prime}=B$, and the previous inequalities remain valid.

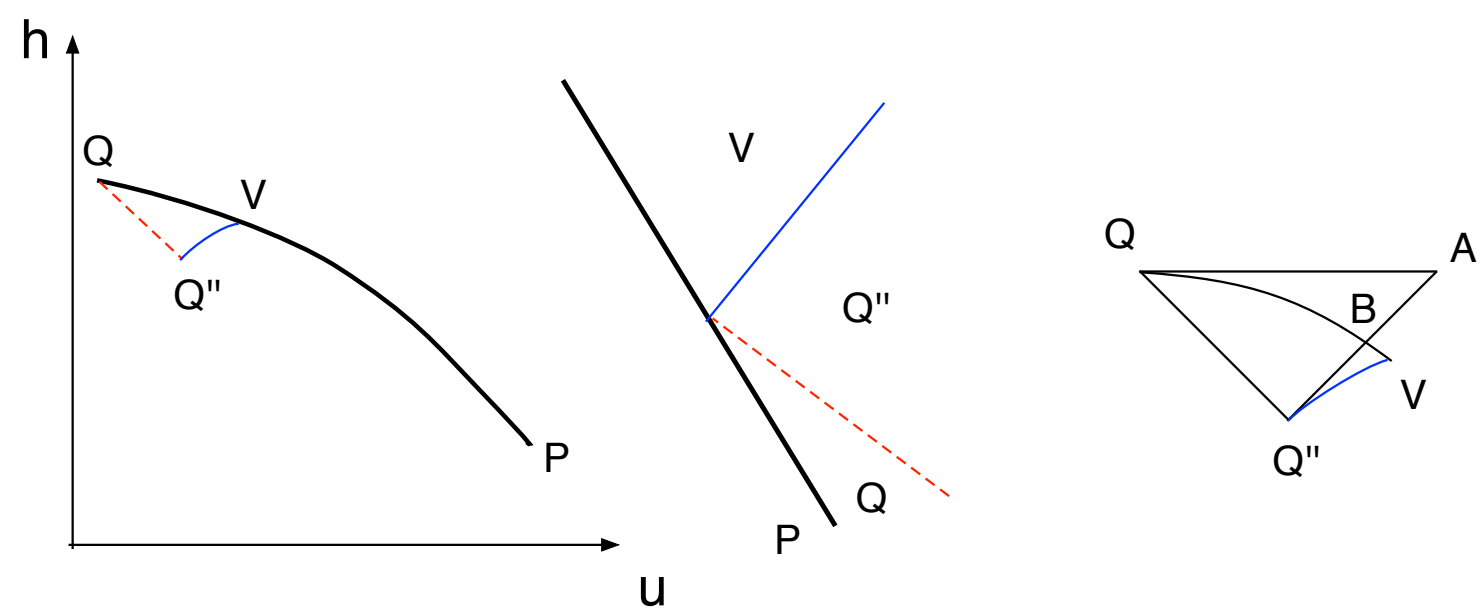

Figure 4: A small 1-rarefaction impinges a large 1-shock. 
CASE 3: The impinging 1-wave is a small rarefaction, connecting $Q$ with a right state $Q^{\prime \prime}$, of strength

$$
\varepsilon=\left(u_{Q^{\prime \prime}}-h_{Q^{\prime \prime}}\right)-\left(u_{Q}-h_{Q}\right) .
$$

In this case the interaction produces an outgoing 2-shock, connecting the states $V$ and $Q^{\prime \prime}$. Here $V$ is the state at the intersection of the 1-shock curve through $P$ and the 2 -shock curve through $Q^{\prime \prime}$. As shown in Fig. 4 , call $A=\left(u_{A}, h_{A}\right)$ the point such hat

$$
u_{A}-h_{A}=u_{Q^{\prime \prime}}-h_{Q^{\prime \prime}}, \quad h_{A}=h_{Q},
$$

and let $B$ be the point at the intersection of the 1-shock curve through $P$ and the segment $A Q^{\prime \prime}$. Recalling that the 2 -shock curve through $Q^{\prime \prime}$ has a second order tangency with the segment $A Q^{\prime \prime}$, and using the inequality

$$
-1 \leq \frac{d h+}{d u_{+}} \leq-\delta_{0}<0
$$

for some $\delta_{0}>0$ sufficiently small, we obtain

$$
\begin{aligned}
& \text { [strength of the outgoing 2-shock }]=\left(u_{V}+h_{V}\right)-\left(u_{Q^{\prime \prime}}+h_{Q^{\prime \prime}}\right) \\
& \quad=\left(u_{B}+h_{B}\right)-\left(u_{Q^{\prime \prime}}+h_{Q^{\prime \prime}}\right)+\mathcal{O}\left(\varepsilon^{3}\right) \\
& \quad \leq\left(1-\frac{\delta_{0}}{2}\right)\left[\left(u_{Q^{\prime \prime}}-h_{Q^{\prime \prime}}\right)-\left(u_{Q}-h_{Q}\right)\right]+\mathcal{O}\left(\varepsilon^{3}\right) \\
& \quad=\left(1-\frac{\delta_{0}}{2}\right) \varepsilon+\mathcal{O}\left(\varepsilon^{3}\right)<\varepsilon=\text { [strength of the incoming 1-rarefaction]. }
\end{aligned}
$$

Together, the above three cases prove part (ii) of Lemma 2.1.

\subsection{Wave measures.}

Let now $x \mapsto(v(x), u(x))$ be any profile with bounded variation. As in Chapter 10 of [4], we can define the signed measures $\mu_{1}, \mu_{2}$ describing strength of waves. Namely, for $i=1,2$,

- The atomic part of $\mu_{i}$ is supported on the countable set of points where $v$ or $u$ have a jump. If $\bar{x}$ is one such point, then $\mu_{i}(\{\bar{x}\})$ is the signed strength of the $i$-th wave in the solution of the Riemann problem with left and right data

$$
(v, u)(\bar{x}-), \quad(v, u)(\bar{x}+) .
$$

- The continuous part of $\mu_{i}$ is defined as the continuous part of the distributional derivative of the scalar function $x \mapsto w_{i}(x)$. Since $w_{i}$ has bounded variation, this is a bounded measure.

These measures can be decomposed into a positive and a negative part, so that

$$
\mu_{i}=\mu_{i}^{+}-\mu_{i}^{-}, \quad\left|\mu_{i}\right|=\mu_{i}^{+}+\mu_{i}^{-} .
$$

Notice that: 
- $\mu_{i}^{+}$accounts for $i$-rarefaction waves.

- The continuous part of $\mu_{i}^{-}$accounts for $i$-compression waves

- The atomic part of $\mu_{i}^{-}$accounts for $i$-shocks.

As shown in [4], Glimm's functionals (originally defined for piecewise constant functions) can be extended to arbitrary BV functions. The total strength of waves is defined as

$$
V \doteq \sum_{i=1,2}\left|\mu_{i}\right|(\mathbb{R})
$$

while the interaction potential is

$$
Q \doteq \int_{x<y} d\left|\mu_{2}\right|(x) d\left|\mu_{1}\right|(y)+\sum_{i=1,2}\left|\mu_{i}\right|(\mathbb{R}) \cdot \mu_{i}^{-}(\mathbb{R}) .
$$

Notice that (2.33) accounts for the product of strengths of all couples of approaching waves. We recall that two waves of the same family are approaching if at least one of them is a compression or a shock.

Next, consider a solution $(v, u)$ of (1.1) defined for $t \in\left[t_{0}, t_{1}\right]$ and let $V(t), Q(t)$ be the total strength of waves and the wave interaction potential at time $t$. As shown in Chapter 10 of [4], these functionals satisfy the same estimates valid for Glimm or front-tracking approximations. In particular, from the interaction estimates in Proposition 2.1 it follows

Lemma 2.2 For any given $K_{0}$ and $a, \varepsilon_{0}>0$, there exists $\delta_{0}>0$ such that the following holds. Assume that

(i) the density remains bounded away from zero: $h(v(t, x)) \geq$ a for all $t \in\left[t_{0}, t_{1}\right], x \in \mathbb{R}$,

(ii) the total strength of waves in the initial data satisfies $V\left(t_{0}\right) \leq K_{0}$, and

(iii) the solution $(v, u)$ does not contain any shock of strength $>\delta_{0}$.

Then the function

$$
t \mapsto V(t)+\varepsilon_{0} Q(t), \quad t \in\left[t_{0}, t_{1}\right]
$$

is non-increasing.

Given a BV solution $U=(v, u)$ of (1.1), we denote by

$$
\lambda_{1}(t, x) \doteq-c(v(t, x)), \quad \lambda_{2}(t, x) \doteq c(v(t, x)),
$$

the two wave speeds at the point $(t, x)$, as in (2.6). Following [14, for $i=1,2$, by a generalized $i$-characteristic we mean an absolutely continuous curve $x=x(t)$ such that

$$
\dot{x}(t) \in\left[\lambda_{i}(t, x+), \lambda_{i}(t, x-)\right]
$$

for a.e. $t$. 
For a given terminal point $\bar{x}$ we shall consider the minimal $i$-characteristic through $\bar{x}$, defined as

$$
\xi(t, \bar{x}) \doteq \min \left\{x(t) ; \quad x \text { is an } i \text {-characteristic, } \quad x\left(t_{1}\right)=\bar{x}\right\} .
$$

As proved in [14], the curve $\xi(\cdot, \bar{x})$ is itself an $i$-characteristic. Indeed, for a.e. $t$ the functions $w_{1}, w_{2}$ and hence also the wave speed $\lambda_{i}$ are continuous at $(t, \xi(t))$. Therefore we can simply write

$$
\dot{\xi}(t)=\lambda_{i}(t, \xi(t))
$$

In addition to the wave measures $\mu_{i}$, one can also introduce a scalar, positive measure $\mu^{\text {int }}$ on the $t$ - $x$ plane bounding the amount of interaction, and hence the production of new waves. More precisely, let $\left(U_{\nu}\right)_{\nu \geq 1}$ be a sequence of piecewise constant front-tracking solutions, converging to the exact $\mathrm{BV}$ solution $U=(v, u)$.

For each $\nu \geq 1$ we can also construct a purely atomic measure $\mu_{\nu}^{\text {int }}$ by setting

$$
\mu_{\nu}^{i n t}(\{\bar{P}\})=\left|\sigma^{\prime} \sigma^{\prime \prime}\right|
$$

for every point $\bar{P}=(\bar{t}, \bar{x})$ at which two incoming fronts interact, with strengths $\sigma^{\prime}, \sigma^{\prime \prime}$ respectively. By taking a suitable subsequence, we can achieve the weak convergence of measures

$$
\mu_{\nu}^{i n t} \rightarrow \mu^{i n t}
$$

for some positive measure $\mu^{\text {int }}$, which we call a measure of wave interaction for the solution $U$. Taking the limit of front tracking approximations one obtains a useful important property of this measure, namely:

Lemma 2.3 For $i \in\{1,2\}$, let $t \mapsto \xi(t)$ and $t \mapsto \tilde{\xi}(t)$ be two minimal $i$-characteristics, with $\xi(t) \leq \tilde{\xi}(t)$ for $t \in\left[t_{0}, t_{1}\right]$. Then one has the estimate

$$
\mu_{i}^{ \pm}\left(\left[\xi\left(t_{1}\right), \tilde{\xi}\left(t_{1}\right)[) \leq \mu_{i}^{ \pm}\left(\left[\xi\left(t_{0}\right), \tilde{\xi}\left(t_{0}\right)[)+C \cdot \mu^{i n t}(\Omega)\right.\right.\right.\right.
$$

where

$$
\Omega \doteq\left\{(t, x) ; \quad t \in\left[t_{0}, t_{1}\right], x \in[\xi(t), \tilde{\xi}(t)[\}\right.
$$

In other words, the total amount of (positive or negative) $i$-waves at time $t_{1}$ contained in the interval $\left[\xi\left(t_{1}\right), \tilde{\xi}\left(t_{1}\right)\right.$ [ can be estimated in terms of the "old $i$-waves" (positive or negative, respectively) present at time $t_{0}$ inside the interval $\left[\xi\left(t_{0}\right), \tilde{\xi}\left(t_{0}\right)[\right.$, plus some "new waves" generated by wave interactions occurring inside the domain $\Omega$ enclosed between the two characteristics. The total strength of these new waves can be bounded in terms of the interaction measure $\mu^{\text {int }}$.

A precise value for the constant $C$ in (2.39) can be determined using the interaction estimates (2.14) -(2.16) . In particular, by taking the limit of front tracking approximations, one obtains

Lemma 2.4 For any given constants $K_{0}, a$ and $\varepsilon_{0}>0$, one can find $\delta_{0}>0$ such that, under the assumptions (i)-(iii) of Lemma [2.2, one has 
(i) the total amount of interaction satisfies

$$
\mu^{i n t}\left(\left[t_{0}, t_{1}\right] \times \mathbb{R}\right) \leq 2 K_{0}^{2},
$$

(ii) the estimate (2.39) holds with $C=\varepsilon_{0}$.

\section{Decay of positive waves.}

Differentiating (2.5) and writing the wave speed as $c=c\left(w_{2}-w_{1}\right)$ with $c$ as in (2.7), one obtains

$$
\left\{\begin{array}{l}
w_{1, x t}-c w_{1, x x}=-c^{\prime} w_{1, x}^{2}+c^{\prime} w_{1, x} w_{2, x}, \\
w_{2, x t}+c w_{2, x x}=c^{\prime} w_{1, x} w_{2, x}-c^{\prime} w_{2, x}^{2} .
\end{array}\right.
$$

The above system would be easy to integrate if we did not have the mixed terms $w_{1, x} w_{2, x}$. To get rid of these terms, we first multiply each equation in (2.5) by a function $\phi=\phi\left(w_{2}-w_{1}\right)$ and then differentiate. For example, the second equation yields

$$
\begin{aligned}
& {\left[\phi w_{2, x}\right]_{t}+c\left[\phi w_{2, x}\right]_{x}} \\
& \quad=\phi\left[c^{\prime} w_{1, x} w_{2, x}-c^{\prime} w_{2, x}^{2}\right]+\phi^{\prime}\left(w_{2, t}-w_{1, t}\right) w_{2, x}+c \phi^{\prime}\left(w_{2, x}-w_{1, x}\right) w_{2, x} \\
& \quad=-\phi\left[-c^{\prime} w_{1, x} w_{2, x}+c_{w_{2}} w_{2, x}^{2}\right]-2 c \phi^{\prime} w_{1, x} w_{2, x} \\
& \quad=-\phi c^{\prime} w_{2, x}^{2}
\end{aligned}
$$

provided that

$$
\frac{\phi^{\prime}}{\phi}=\frac{c^{\prime}}{2 c}=\frac{1}{2} \frac{\gamma+1}{\gamma-1} \frac{1}{w_{2}-w_{1}} .
$$

Computing an explicit solution of (3.3) we find

$$
\phi\left(w_{2}-w_{1}\right)=\left(w_{2}-w_{1}\right)^{\frac{\gamma+1}{2 \gamma-2}}=(2 h)^{\frac{\gamma+1}{2 \gamma-2}} .
$$

In the end, this yields a decay estimate along any 2-characteristic $t \mapsto x(t)$.

$$
\frac{d}{d t}\left(\phi w_{2, x}\right)(t, x(t))=-\phi c_{w_{2}} w_{2, x}^{2} \leq-C_{2} w_{2, x}^{2},
$$

for some constant $C_{2}>0$ depending only on the upper and lower bounds for the density. Of course, an entirely similar estimate holds for 1-rarefactions.

Next, let $\xi_{1}(t)<\xi_{2}(t)$ be two 2-characteristics. Calling $c=c\left(w_{2}-w_{1}\right)$ the characteristics speed as a function of the Riemann coordinates, we have

$$
\frac{d}{d t}\left(\xi_{2}(t)-\xi_{2}(t)\right)=\dot{\xi}_{2}(t)-\dot{\xi}_{1}(t)=\int_{\xi_{1}(t)}^{\xi_{2}(t)} c^{\prime}\left(w_{2, x}-w_{1, x}\right) d x .
$$

Notice that the above identity involves also the 1-waves inside the interval $\left[\xi_{1}(t), \xi_{2}(t)\right]$. We seek an equivalent way to express the distance between two characteristics, which does not involve the contribution of intermediate 1-waves. Toward this goal, consider the integral

$$
Z(t) \doteq \int_{\xi_{1}(t)}^{\xi_{2}(t)} \varphi d x
$$


where $\varphi=\varphi\left(w_{2}-w_{1}\right)=\left(w_{2}-w_{1}\right)^{-\frac{\gamma+1}{2 \gamma-2}}$ which satisfies $c^{\prime} \varphi=-2 c \varphi^{\prime}$. We compute

$$
\begin{aligned}
\frac{d}{d t} Z(t) & =\dot{\xi}_{2} \varphi\left(\xi_{2}\right)-\dot{\xi}_{1} \varphi\left(\xi_{1}\right)+\int_{\xi_{1}}^{\xi_{2}} \varphi^{\prime}\left(w_{2, t}-w_{1, t}\right) d x \\
& =c\left(\xi_{2}\right) \varphi\left(\xi_{2}\right)-c\left(\xi_{1}\right) \varphi\left(\xi_{1}\right)-\int_{\xi_{1}}^{\xi_{2}} \varphi^{\prime} c\left(w_{2, x}+w_{1, x}\right) d x \\
& =\int_{\xi_{1}}^{\xi_{2}}\left[c^{\prime}\left(w_{2, x}-w_{1, x}\right) \varphi+c \varphi^{\prime}\left(w_{2, x}-w_{1, x}\right)\right] d x-\int_{\xi_{1}}^{\xi_{2}} \varphi^{\prime} c\left(w_{2, x}+w_{1, x}\right) d x \\
& =\int_{\xi_{1}}^{\xi_{2}}\left[-2 c \varphi^{\prime}\left(w_{2, x}-w_{1, x}\right)+c \varphi^{\prime}\left(w_{2, x}-w_{1, x}\right)\right] d x-\int_{\xi_{1}}^{\xi_{2}} \varphi^{\prime} c\left(w_{2, x}+w_{1, x}\right) d x \\
& =\int_{\xi_{1}}^{\xi_{2}} c^{\prime} \varphi w_{2, x} d x .
\end{aligned}
$$

Notice that the last two equalities were obtained using the identity $c^{\prime} \varphi=-2 c \varphi^{\prime}$, which produces a cancellation of all terms involving 1-waves.

\section{A periodic interaction pattern}

As a preliminary to the blow-up example, in this section we construct a piecewise constant approximate solution with a periodic interaction pattern.

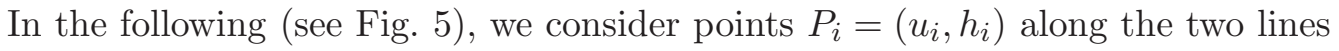

$$
\gamma_{0}=\{(u, h) ; \quad h>0, \quad u-h=0\}, \quad \gamma_{1}=\{(u, h) ; \quad h>0, \quad u-h=1\} .
$$

Lemma 2. There exists a point $P_{0} \in \gamma_{0}$ such that the following holds (Fig. 5, right). Consider the point $P_{1}=\left(u_{0}+\frac{1}{2}, h_{0}-\frac{1}{2}\right) \in \gamma_{1}$. Let $P_{4} \in \gamma_{0}$ be the point along the 1-shock curve through $P_{1}$ and let $P_{2} \in \gamma_{1}$ be the point along the 2-shock curve through $P_{4}$. Finally, call $P_{3}=\left(u_{2}-\frac{1}{2}, h_{2}+\frac{1}{2}\right) \in \gamma_{0}$ and let $P_{5} \in \gamma_{1}$ be the point along the 1-shock curve through $P_{3}$. Then $h_{5}<h_{0}$.

As a consequence, there is a left state $L$ which can be connected to both $P_{0}$ and $P_{5}$ by 1-shocks.

Proof. First, consider the 1-shock through $P=\left(\frac{1}{2}, \frac{1}{2}\right)$. This intersects the line $\gamma_{1}$ at some point $Q$, say with $h(Q)=\kappa>0$.

Next, for $\varepsilon>0$ small consider the points

$$
P_{0}=\left(\frac{1}{2}+\varepsilon, \frac{1}{2}+\varepsilon\right), \quad P_{1}=(1+\varepsilon, \varepsilon) .
$$

Starting from $P_{1}$, construct the corresponding point $P_{4}$ and then $P_{2}=(1+\eta(\varepsilon), \eta(\varepsilon))$. By (2.25) and the boundedness of the amplification factor $a(\theta)$, as $\varepsilon \rightarrow 0+$ we also have $\eta(\varepsilon) \rightarrow 0$.

Finally, call $P_{3}=(1+\eta(\varepsilon), 1+\eta(\varepsilon))$ and let $P_{5} \in \gamma_{1}$ be the point along the 1 -shock curve through $P_{3}$. 


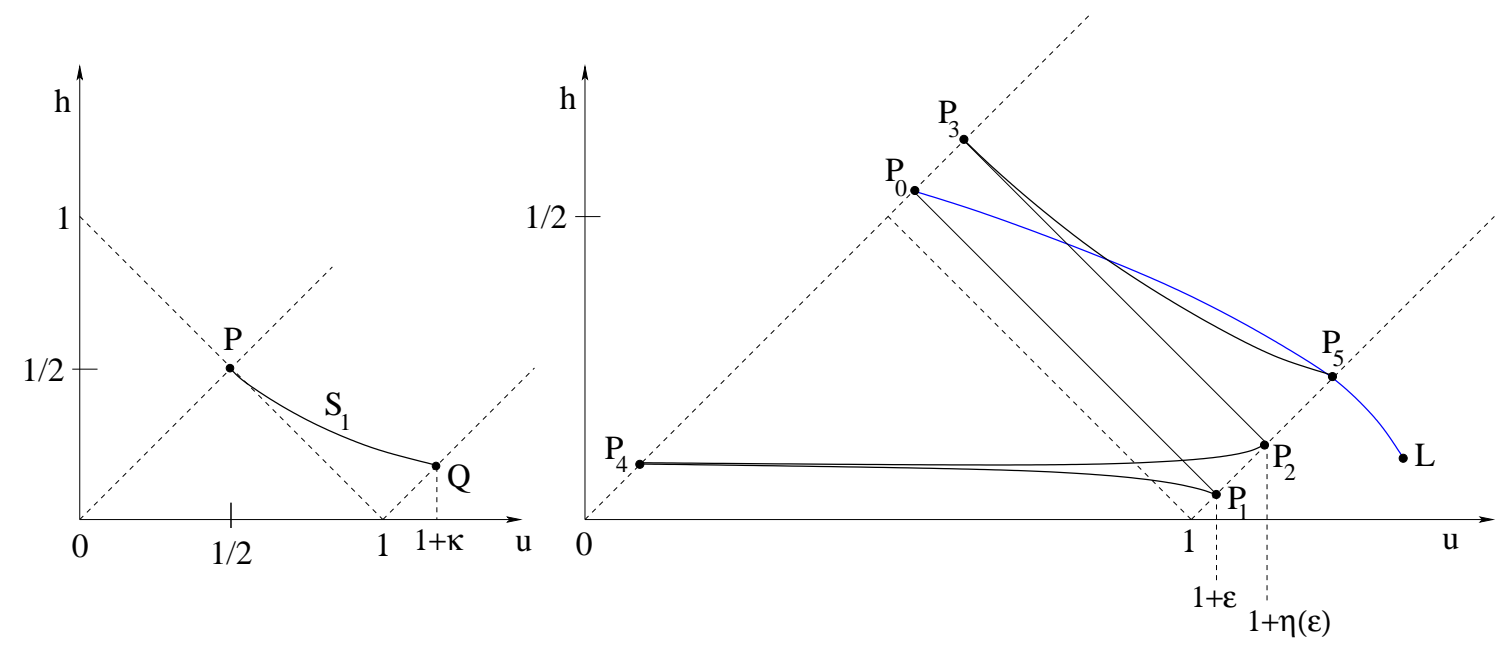

Figure 5: Left: the 1-shock curve through $\left(\frac{1}{2}, \frac{1}{2}\right)$. Right: the various points $P_{i}$ considered in Lemma 1.

By (2.30), it follows

$$
h_{3}-h_{5}>h(P)-h(Q)=\frac{1}{2}-\kappa
$$

Therefore

$$
\begin{aligned}
h_{0}- & h_{5}=\left(h_{0}-h_{3}\right)+\left(h_{3}-h_{5}\right) \\
& =\left(h_{1}-h_{2}\right)+\left(h_{3}-h_{5}\right)>\varepsilon-\eta(\varepsilon)+\left(\frac{1}{2}-\kappa\right)>0
\end{aligned}
$$

for $\varepsilon>0$ small enough. We thus have

$$
h_{5}<h_{0}<h_{3}, \quad u_{0}<u_{3}<u_{5} .
$$

This proves the first statement in Lemma 2.

It remains to prove that there exists a left state $L=\left(u_{L}, \rho_{L}\right)$ which is connected to both $P_{5}$ and $P_{0}$ by a 1-shock. Referring to Fig. 66 consider the 1-shock curve through $P_{5}$. Let $Q=\left(u_{Q}, h_{Q}\right)$ be any point on this curve. Notice that, as $u_{Q} \rightarrow+\infty$, we have $h_{Q} \rightarrow 0$.

Next, let $\bar{P}(Q)=(\bar{u}, \bar{h})$ be the point where the 1-shock curve through $Q$ intersects the line $\gamma_{0}=\{h-u=0\}$. Since this shock curve is concave down, one has

$$
\liminf _{u_{Q} \rightarrow+\infty} \frac{h_{5}-\bar{h}}{u_{5}-\bar{u}} \geq \lim _{u_{Q} \rightarrow+\infty} \frac{h_{Q}-h_{5}}{u_{Q}-u_{5}}=0 .
$$

Therefore, as $u_{Q} \rightarrow+\infty$ the point $\bar{P}(Q)$ approaches the point $\left(u_{5}-1, h_{5}\right)$. On the other hand, as $u_{Q} \rightarrow u_{5}$ one has $\bar{P}(Q) \rightarrow P_{3}$. Since $h_{0}>h_{5}$, by continuity, there is some choice of $Q$ such that $\bar{P}(Q)=P_{3}$. This completes the proof of Lemma 2 .

Using Lemma 1 we now construct a front tracking solution to the system (1.1) with a periodic interaction pattern. Referring to Fig. 7, at time $t=\tau$ the piecewise constant solution $(u, h)(\tau, \cdot)$ takes the values $L, P_{5}, P_{2}, P_{3}, P_{4}$. As time increases, the following interactions take place, one after the other.

(i) The 2-rarefaction $P_{1} P_{2}$ crosses the 1-compression $P_{2} P_{3}$. Afterwards, this 1-compression breaks into a 1-shock and a 2-rarefaction. In the end, the states $P_{1}$ and $P_{3}$ are connected by the 1 -shock $P_{1} P_{4}$ followed by the 2 -rarefaction $P_{4} P_{3}$. 




Figure 6: Constructing the left state $L$.

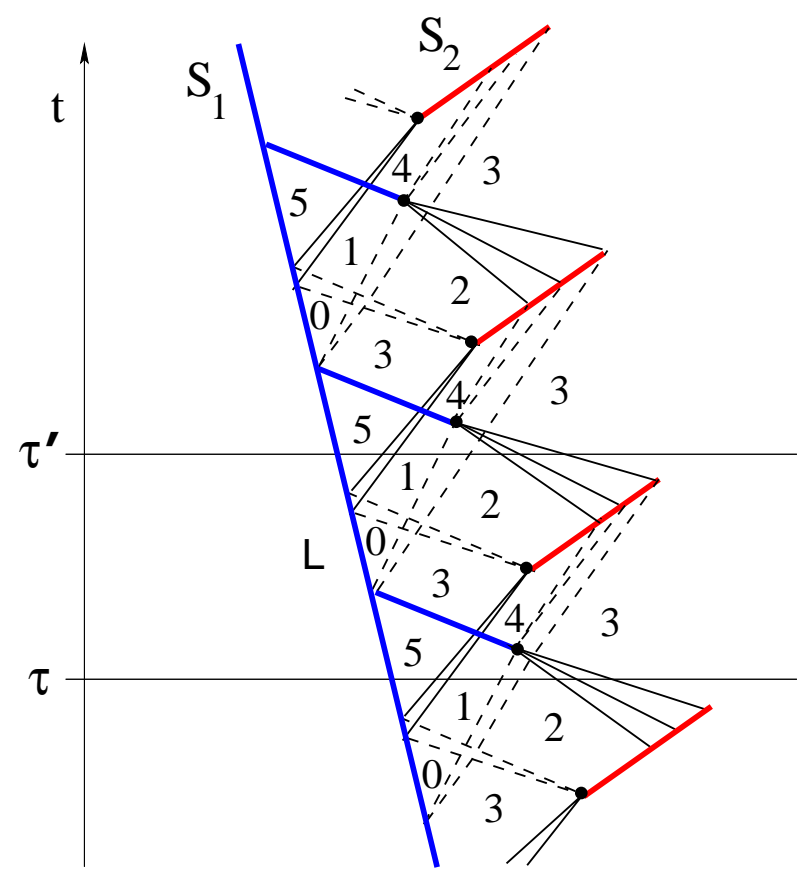

Figure 7: A periodic interaction pattern in the $(x, t)$-plane. Here $0,1, \ldots, 5$ and $L$ refer to the states $P_{0}, P_{1}, \ldots, P_{5}$ and to the left state $L$ considered in Lemma 1 . The thick solid lines are shocks, the thin solid lines represent compressions, while the dashed lines are rarefaction fronts. 
(ii) The 2-compression $P_{5} P_{1}$ crosses the 1-shock $P_{1} P_{4}$. The Riemann problem is solved by the 1-shock $P_{5} P_{3}$ and the 2-compression $P_{3} P_{4}$.

(iii) The 1-shock $P_{5} P_{3}$ hits the 1 -shock $L P_{5}$, generating the 1 -shock $L P_{0}$ and the 2 -rarefaction $P_{0} P_{3}$.

(iv) The 2-compression $P_{3} P_{4}$ breaks into the 1-rarefaction $P_{3} P_{2}$ and the 2-shock $P_{2} P_{4}$.

(v) The 2-rarefaction $P_{0} P_{3}$ crosses the 1-rarefaction $P_{3} P_{2}$, producing the 1-rarefaction $P_{0} P_{1}$ and the 2-rarefaction $P_{1} P_{2}$.

(vi) The 1-rarefaction $P_{0} P_{1}$ hits the 1 -shock $L P_{0}$, producing the 1 -shock $L P_{5}$ and the the 2-compression $P_{5} P_{1}$.

(vii) The 2-shock $P_{2} P_{4}$ is canceled by the 2-rarefaction $P_{4} P_{3}$, producing the 1 -compression $P_{2} P_{3}$.

At time $t=\tau^{\prime}$ we have reached the same configuration as at time $\tau$, and the periodic pattern can be continued.

Remark. If the initial data had small total variation, then the standard wave interaction estimates [4, 19, 29] would imply that the interaction potential approaches zero. As proved in 25], the solution would converge to the solution of the Riemann Problem with left and right data $\left(L, P_{3}\right)$. In the present interaction pattern, however, this does not happen because wave strengths are large. In particular, notice that the 2-compression $P_{5} P_{1}$ is greatly amplified when it crosses the large 1-shock $P_{1} P_{4}$.

\section{An example with finite time blow-up of the total variation}

In this section we provide an affirmative answer to the question ( $\mathbf{Q})$ considered in the Introduction. Namely, we construct a piecewise smooth approximate solution of (1.1) such that:

(C1) At each interaction, the strengths of outgoing waves is the same as in an exact solution.

(C2) For some constant $C_{0}>0$, all rarefaction waves satisfy a decay estimate of the form

$$
\frac{d}{d t}\left(\phi w_{i, x}\right)\left(t, x_{i}(t)\right) \leq-C_{0} w_{i, x}^{2}, \quad i=1,2 .
$$

Here $\varphi$ is the function at (3.4) and $t \mapsto x_{i}(t)$ is any $i$-characteristic.

(C3) The density $\rho$ remains uniformly positive.

(C4) The total variation blows up in finite time. 

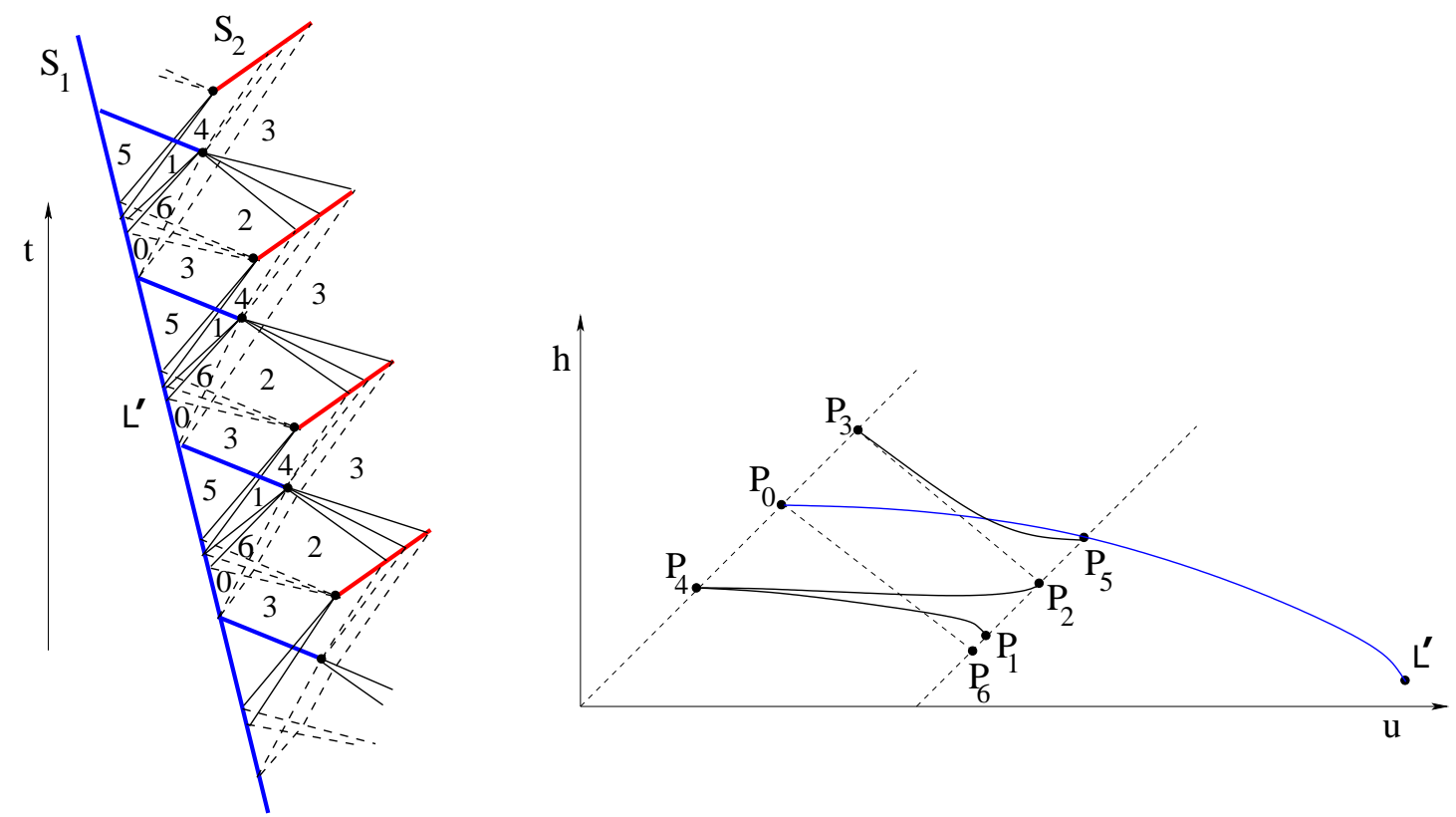

Figure 8: Left: a modified periodic pattern. Compared with the pattern in Fig. 7 the compression wave joining $P_{5}$ to $P_{1}$ is now split in two parts. This creates an additional state, which we call $P_{6}$. Right: the location of the new states $P_{0}, \ldots, P_{6}$ and $L^{\prime}$, in the $u$-h plane.

\subsection{Outline of the construction}

Then to construct an approximate solution of (1.1) whose total variation blows up in finite time, the periodic pattern constructed in the previous section will be modified in two ways:

(i) By slightly changing the wave speeds, the interaction pattern can be repeated on a sequence of shorter and shorter time intervals $\left[\tau_{n-1}, \tau_{n}\right]$, with $\lim _{n \rightarrow \infty} \tau_{n}=T<\infty$.

(ii) In the original pattern the 2-shock connecting $P_{2}$ with $P_{4}$ is entirely cancelled by the 2-rarefaction connecting $P_{4}$ with $P_{3}$. We slightly change the speeds of these two waves so that they do not entirely cancel each other. More precisely, for every $n$ large enough, at the terminal time $T$ the solution will still contain a 2-shock and a 2-rarefaction, both of strength $\alpha n^{-1}$, connecting the states $P_{3}^{(n+1)}$ and $P_{3}^{(n)}$. Here $\alpha$ is a fixed positive constant. These are the remaining portions of the 1-shock and 1-rarefaction which are not completely cancelled by the $n$-th iteration of the basic pattern. The total strength of all these waves is $\sum_{n \geq N} 2 \alpha n^{-1}=+\infty$, providing the blow-up of the total variation as $t \rightarrow T-$.

Because of (ii), it is clear that the intermediate states $P_{0}, \ldots P_{5}$ generated by this interaction pattern can no longer repeat cyclically, but will slightly change after each round of interactions. Still, as $t \rightarrow T$, a periodic interaction pattern will be approached. The construction of the approximate solution will be achieved in the next three steps. 


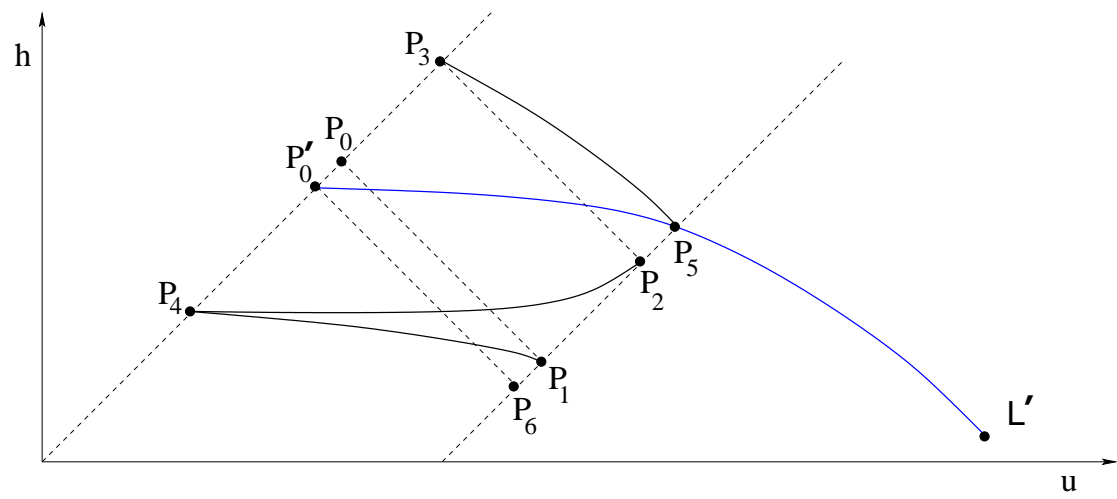

Figure 9: Constructing a perturbed periodic pattern. Here the states $P_{0}, P_{1}, \ldots, P_{5}$ are the same as in Fig. 7 The states $P_{0}^{\prime}, P_{1}, \ldots, P_{6}$ and $L^{\prime}$ are those in the new pattern shown in Fig. 8

\subsection{A perturbed periodic interaction pattern.}

To construct our approximate solution, we begin by defining a slightly different periodic interaction pattern. As shown in Fig. 8, the compression wave between the states $P_{1}$ and $P_{5}$ is now split in two parts.

- A small portion breaks at the same point where the 1-compression waves merge into a large 1-shock. This portion is thus completely cancelled by the interaction.

- The remaining portion eventually forms a large 2-shock, as in the previous periodic pattern.

As a result, the new periodic approximate solution will contain an additional constant state $P_{6}$ between the two portions of this 2 -compression wave. Notice that, as $P_{6} \rightarrow P_{1}$, the new pattern becomes identical to the old one. Being able to partition the 2-compression into two separate waves adds one more degree of freedom to the construction of a periodic pattern. This will be used to achieve more easily a convergence estimate.

A periodic pattern as in Fig. 8, can be obtained by a perturbation argument, starting with the pattern constructed in the previous section, and using the implicit function theorem. Our construction is better explained with the aid of Fig. 9.

We start from our earlier periodic example in Fig. 5 including states $P_{0}, \ldots, P_{5}$ and $L$. Furthermore, by the proof of Lemma 2, we have

$$
h\left(P_{0}\right)>h\left(P_{5}\right) .
$$

We can thus find a state $P_{0}^{\prime}$ along the line segment $P_{4} P_{0}$, such that

$$
h\left(P_{0}^{\prime}\right)>h\left(P_{5}\right) .
$$

Note that $P_{0}^{\prime}$ can be chosen arbitrarily close to $P_{0}$,

We then call $P_{6}$ the intersection between the 1 -wave curve through $P_{0}^{\prime}$ and the 2 -wave curve through $P_{1}$. Notice that the 2 -wave with left state $P_{1}$ and right state $P_{6}$ is a compression wave. 

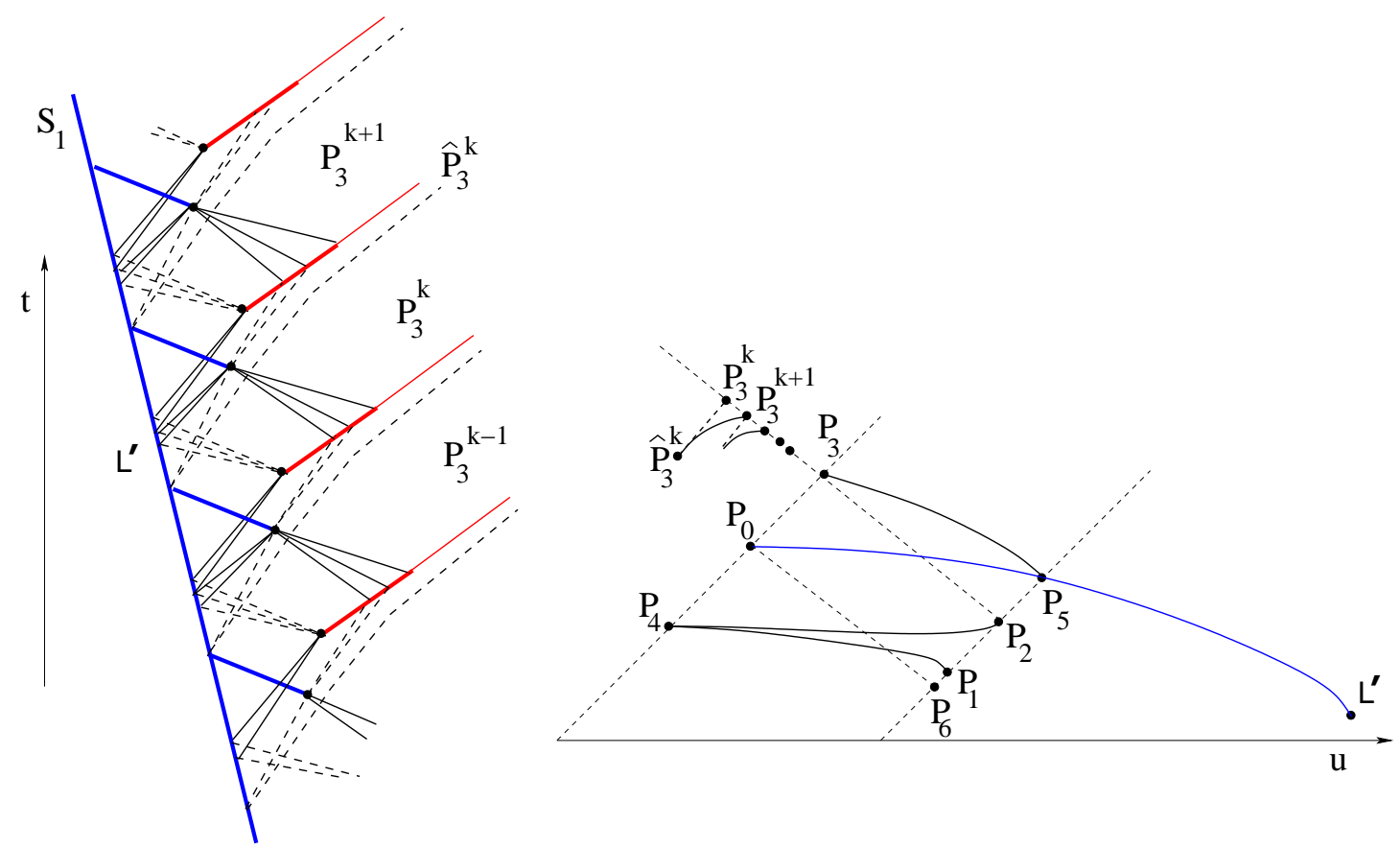

Figure 10: Left: by slightly perturbing the periodic pattern in Fig. 8 , we obtain a new pattern where, at the $k$-th iteration, an additional pair of shock and rarefaction waves is produced, each with strength $\alpha / k$. Right: the sequence of left, middle, and right states $P_{3}^{k+1}, \widehat{P}_{3}^{k}$, and $P_{3}^{k}$.

Finally, by (5.2) and using the same argument as in the proof of Lemma 2, we can find a new left state $L^{\prime}$ which is connected to both $P_{0}^{\prime}$ and $P_{5}$ by a 1 -shock.

One now checks that the states $P_{0}^{\prime}, P_{1}, \ldots, P_{6}$ and $L^{\prime}$ produce the periodic pattern in Fig. 9 . In particular, notice that the Riemann problem with left and right states $P_{1}, P_{3}$ is still solved by the 1 -shock $P_{1} P_{4}$ and the 2 -rarefaction $P_{4} P_{3}$.

\subsection{A sequence of nearly periodic patterns.}

Next, we slightly modify the previous periodic interaction pattern by assuming that, at the $k$-th iteration, the 2 -shock $P_{2} P_{4}$ is not entirely cancelled by the 2-rarefaction $P_{4} P_{3}$. Instead, a pair of 2-waves survive, namely (see Fig 10):

- a 2-shock of strength $\alpha / k$, joining the left state $P_{3}^{k+1}$ with an intermediate state $\widehat{P}_{3}^{k}$,

- a 2-rarefaction, also of strength $\alpha / k$, joining the intermediate state $\widehat{P}_{3}^{k}$ to the right state $P_{3}^{k}$.

Since we require that these two shock and rarefaction waves have exactly the same strength (measured in Riemann invariants), all states $P_{3}^{k}, k=1,2, \ldots$ must lie along the same 1-wave curve through $P_{3}$. Recalling that shock and rarefactions curves coincide up to second order [4, 29], for some constant $C$ we have

$$
\left|P_{3}^{k+1}-P_{3}^{k}\right| \leq \frac{C}{k^{3}}
$$


Therefore, by choosing $\alpha>0$ small enough, we can uniquely determine the states $P_{3}^{k}$ so that

$$
P_{3}^{k} \rightarrow P_{3} \quad \text { as } k \rightarrow \infty .
$$

In turn, we claim that all other intermediate states $P_{i}^{k}$, with $i \in\{0,1,2,4,5,6\}$ and $k \geq 1$, can be uniquely determined as well. Indeed, these can be constructed in the following order:

$$
P_{3}^{k} \rightarrow P_{5}^{k} \rightarrow P_{2}^{k} \rightarrow P_{0}^{k} \rightarrow P_{4}^{k} \rightarrow P_{1}^{k} \rightarrow P_{6}^{k}
$$

- $P_{5}^{k}$ is the state at the intersection of the 1 -shock curve with right state $P_{3}^{(k)}$ and the 1-shock curve through $L^{\prime}$.

- $P_{2}^{k}$ is the state at intersection of the 1 -wave curve through $P_{3}$ and the 2-wave curve through $P_{5}^{k}$.

- $P_{0}^{k}$ is the state at the intersection of the 2-wave curve through $P_{3}^{k}$ and the 1-shock curve through $L^{\prime}$.

- $P_{4}^{k}$ is the state at the intersection of the 2 -shock curve with right state $P_{2}^{k+1}$ and the 2-wave curve through $P_{3}^{k}$.

- $P_{1}^{k}$ is the state at the intersection between the 2-wave curve through $P_{5}^{k}$ and the 1 -shock curve with right state $P_{4}^{k}$.

- Finally, $P_{6}^{k}$ is the state at the intersection between the 1 -wave curve through $P_{0}^{k}$ and the 2 -wave curve through $P_{1}^{k+1}$.

We observe that, by choosing $\alpha>0$ small, all points $P_{3}^{k}$ will lie in a suitably small neighborhood of $P_{3}$. By the implicit function theorem, all the states $P_{5}^{k}, k \geq 1$, are well defined and lie in a suitably small neighborhood of $P_{5}$.

In turn, again by the implicit function theorem, it follows that all the states $P_{2}^{k}$ are well defined and lie in a small neighborhood of $P_{2}$.

After six steps, all sequences of points $P_{3}^{k}, P_{5}^{k}, P_{2}^{k}, P_{0}^{k}, P_{4}^{k}, P_{1}^{k}, P_{6}^{k}, k=1,2, \ldots$ are thus uniquely determined, provided that $\alpha>0$ was chosen sufficiently small. Moreover, we have the convergence

$$
\lim _{k \rightarrow \infty} P_{i}^{k}=P_{i} \quad i=0,1, \ldots, 6 .
$$

We remark that, by the convergence $P_{1}^{k} \rightarrow P_{1}$ and $P_{6}^{k} \rightarrow P_{6}$, it follows that (by possibly shrinking the value of $\alpha$ ) the states $P_{6}^{k}$ and $P_{1}^{k+1}$ are always connected by a 2 -compression (not a 2-rarefaction).

The previous analysis achieves the construction of the modified interaction pattern shown in Fig. 10.

\subsection{An approximate solution with finite time BV blow-up.}

The approximate solution constructed in the previous step (Fig. 10) contains a sequence of 2 -shocks followed by a 2-rarefaction, both of strength $\alpha / k, k=1,2, \ldots$. Clearly, the total strength of all these waves is infinite. 

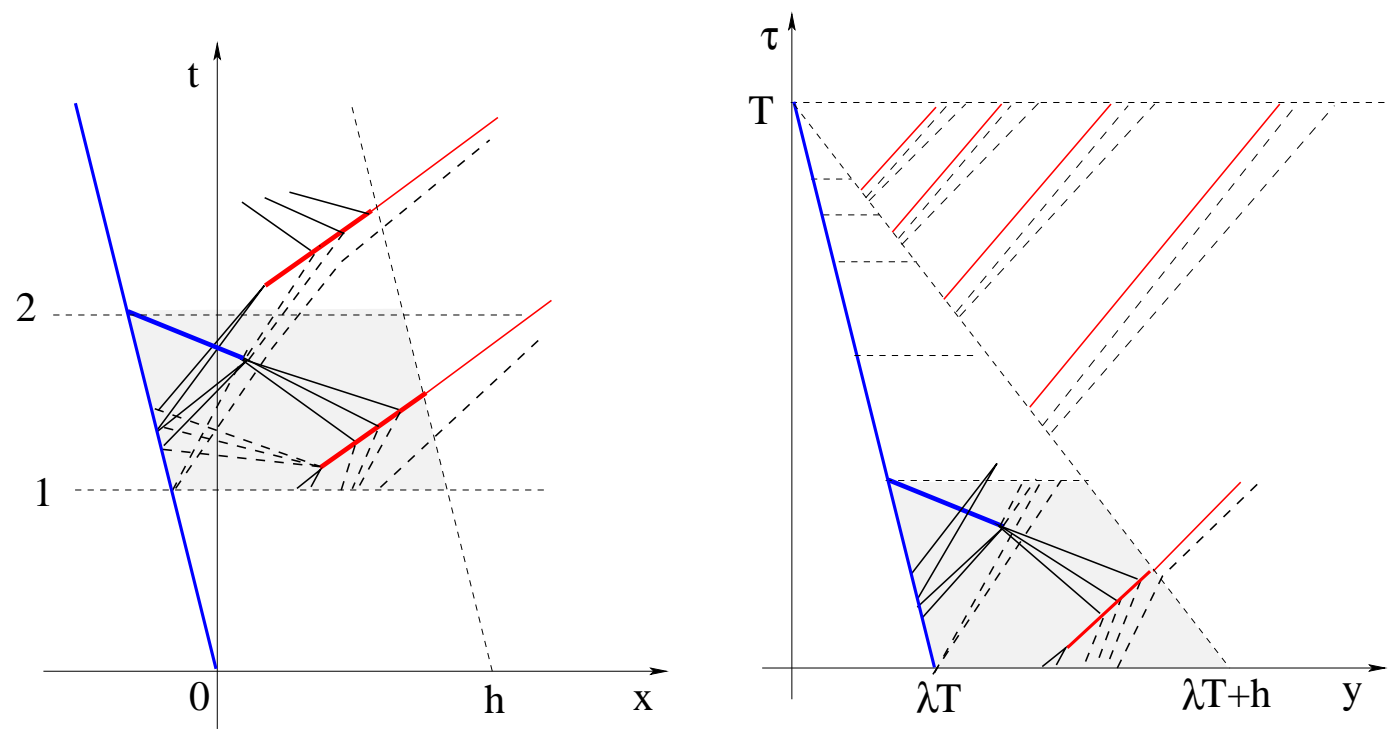

Figure 11: After the transformation of the $t-x$ coordinates defined at (5.6), from the interaction pattern shown on the left (same as the one in Fig. 10) we obtain a new approximate solution where all wave interactions take place within the time interval $[0, T]$. At the terminal time $\tau=T$, the total variation becomes infinite.

To provide an example where blow up of the total variation occurs in finite time, it suffices to slightly modify the wave speeds, so that the interaction cycles repeat over shorter and shorter time intervals $\left[\tau_{k}, \tau_{k+1}\right]$, with $\tau_{k} \rightarrow T$ as $k \rightarrow \infty$.

To fix the ideas, assume that in the previous construction the basic interaction cycle takes place on the parallelograms

$$
\Gamma_{k}=\{(t, x) ; t \in[k, k+1], \quad x \in[-\lambda t,-\lambda t+h]\}, \quad k=1,2, \ldots
$$

for some $h>0$ (see Fig. 11, left). Fix $T>0$ sufficiently large and define

$$
\varepsilon \doteq-\ln \left(1-\frac{1}{T}\right)=\ln \left(1+\frac{1}{T-1}\right) .
$$

Observe that, as $T \rightarrow+\infty$, we have

$$
\varepsilon T=1+\mathcal{O}(1) \cdot T^{-1} .
$$

Consider the transformation (see Fig. 11)

$$
\tau=\left(1-e^{-\varepsilon t}\right) T, \quad y=(x+\lambda t+\lambda T) e^{-\varepsilon t},
$$

defined for $t \geq 0, x \in \mathbb{R}$.

If now $x=\xi(t)$ is the equation of a wave front in the $t$-x coordinates, let $y=\zeta(\tau)$ be the corresponding equation in the $\tau-y$ coordinates. Differentiating w.r.t. $t$ the identity

$$
y(t, \xi(t))=\zeta(\tau(t, \xi(t)))
$$


we compute

$$
\begin{aligned}
\zeta^{\prime}(\tau) & =\frac{y_{t}+y_{x} \xi^{\prime}}{\tau_{t}+\tau_{x} \xi^{\prime}(t)}=\frac{\lambda e^{-\varepsilon t}-(x+\lambda t+\lambda T) \varepsilon e^{-\varepsilon t}+e^{-\varepsilon t} \phi^{\prime}}{T \varepsilon e^{-\varepsilon t}} \\
& =\frac{\lambda(1-\varepsilon T)+\varepsilon(x+\lambda t)+\xi^{\prime}(t)}{\varepsilon T}=\xi^{\prime}(t)+\mathcal{O}(1) \cdot T^{-1},
\end{aligned}
$$

as long as $x+\lambda t \in[0, h]$. In other words, by choosing the blow up time $T$ large enough, the speeds of all waves contained in the strip $\{x+\lambda t \in[0, h]\}$ are almost unchanged by the coordinate transformation. Furthermore, we impose that each pair of 2-shocks and 2rarefactions (created at each interaction cycle) travels with the same speed in the $\tau-y$ as in the old $t$ - $x$ coordinates (see Fig. 11), right).

In view of (5.7), this approximate solution in the $\tau-y$ variables satisfies all conditions (C1)(C4) stated at the beginning of this section.

\section{A necessary condition for blowup}

In the second part of this paper, we prove that, if the total variation blows up in finite time, then the solution must contain an infinite number of large shocks in a neighborhood of some point $\bar{P}=(T, \bar{x})$.

Since the p-system with $p(v)=v^{-\gamma}$ admits a group of rescalings, a precise meaning of "large shock" must be given in terms of the upper and lower bounds on the gas density $\rho$. We recall that, as proved in [21], for any $b>0$ the domain

$$
\mathcal{D}_{b} \doteq\{(h, u) ; \quad h \geq 0, \quad|u| \leq b-h\}
$$

is positively invariant for the system (1.1), with $p$ and $h$ as in (2.1), (2.3). In the following we shall assume that the density $\rho$ remains uniformly positive, and hence the same holds for $h$. We thus consider a solution taking values in the domain

$$
\mathcal{D}_{a b} \doteq\{(h, u) ; \quad|u| \leq b-h, \quad h \geq a\},
$$

for some $0<a<b$. In the following, the total variation of the vector-valued function $x \mapsto(h(t, x), u(t, x))$ on an (possibly unbounded) interval $I \subset \mathbb{R}$ is defined as

$$
\text { Tot.Var. }\{(h, u)(t, \cdot) ; \quad I\} \doteq \sup \sum_{i}\left(\left|h\left(t, x_{i}\right)-h\left(t, x_{i-1}\right)\right|+\left|u\left(t, x_{i}\right)-u\left(t, x_{i-1}\right)\right|\right),
$$

where the supremum is taken over all finite increasing sequences of points $x_{0}<x_{1}<\cdots<x_{N}$ contained in $I$.

Observe that, as long as the solution takes values inside the compact domain $\mathcal{D}_{a b}$, a bound on the total variation of $(v, u)$ is equivalent to a bound on the total variation of $(h(v), u)$. In turn, this is also equivalent to a bound on the total strength of waves, measured in Riemann invariants, as in (2.9).

Theorem 6.1 For any two constants $b>a>0$, there exists $\delta_{0}>0$ such that the following holds. Consider an entropy weak solution $(v, u)$ of (1.1) such that $(h(v), u) \in \mathcal{D}_{a b}$ for all $t, x$, 
and assume that the total variation is initially bounded but blows up at a finite time $T$. Then there exists a point $\bar{x}$ such that every neighborhood of $(T, \bar{x})$ in the $t$-x plane contains infinitely many shocks with strength $\geq \delta_{0}$.

A proof of this theorem will be completed in the next two sections. We observe that, since the initial data $(\bar{v}, \bar{u})$ have bounded variation, for every $\varepsilon_{0}>0$ there exists $R_{0}>0$ sufficiently large such that

$$
\begin{gathered}
\text { Tot.Var. }\{(\bar{v}, \bar{u}) ; \quad]-\infty,-R_{0}[\}<\varepsilon_{0} . \\
\text { Tot.Var. }\{(\bar{v}, \bar{u}) ; \quad] R_{0},+\infty[\}<\varepsilon_{0},
\end{gathered}
$$

For a solution taking values in the domain $\mathcal{D}_{a b}$, the characteristic speeds $\pm c$ in remain uniformly bounded above and below. Indeed, since $a \leq h \leq b$, by (2.6) and (2.3) it follows

$$
c=\sqrt{-p^{\prime}(v)}=\sqrt{A / \gamma} \cdot v^{-(\gamma+1) / 2} \leq \sqrt{A / \gamma} \cdot(b / B)^{(\gamma+1) /(\gamma-1)} \doteq \hat{\lambda} .
$$

By choosing $\varepsilon_{0}$ small enough, by the Glimm interaction estimates it follows that for any $t>0$ the total variation of the solution on the two domains

$$
]-\infty,-R_{0}-\hat{\lambda} t[, \quad] R_{0}+\hat{\lambda} t,+\infty[,
$$

remains uniformly small. Here $\hat{\lambda}$ is the upper bound on all characteristic speed, computed at (6.3). Hence, if the total variation blows up at time $T$, this must happen within the compact interval $\left[-R_{0}-\hat{\lambda} T, R_{0}+\hat{\lambda} T\right]$.

We conclude this section with a preliminary lemma.

Lemma 6.1 For any $B V$ function $x \mapsto(v(x), u(x))$ with

$$
(h(v(x)), u(x)) \in \mathcal{D}_{a b} \quad \text { for all } x \in \mathbb{R},
$$

the following holds.

(i) For any compact interval I, one has

[total strength of all waves in $I] \leq 2(b+[$ total strength of rarefaction waves in $I])$.

(ii) There exists $\delta_{1}>0$ such that, for every subinterval $J \subset \mathbb{R}$ of length $\leq \delta_{1}$ one has

$$
\text { [total strength of all waves in } J] \leq 2 b \text {. }
$$

Proof. To prove (i), call $\mu_{1}, \mu_{2}$ the corresponding wave measures, defined as in Section 2.5. Moreover, call $\mu_{i}^{+}$the positive part of $\mu_{i}$. Then (6.6) means that

$$
\left|\mu_{1}\right|(I)+\left|\mu_{2}\right|(I) \leq 2\left(b+\mu_{1}^{+}(I)+\mu_{2}^{+}(I)\right) .
$$

To prove (6.8) we observe that the $u$ component has a downward jump at every point of shock. More precisely, for an $i$-shock located at a point $\bar{x}$, one has

$$
u(\bar{x}+)-u(\bar{x}-) \leq w_{i}(\bar{x}+)-w_{i}(\bar{x}-)<0 .
$$


If $I=[\alpha, \beta]$, since $u$ takes values inside $\mathcal{D}_{a b}$ we have the inequalities

$$
\begin{gathered}
-2 b \leq u(\beta+)-u(\alpha-) \leq \mu_{1}(I)+\mu_{2}(I), \\
\left|\mu_{1}\right|(I)-2 \mu_{1}^{+}(I)+\left|\mu_{2}\right|(I)-2 \mu_{2}^{+}(I) \leq 2 b .
\end{gathered}
$$

This yields (6.8).

Next, if (ii) fails, then we can find a sequence of intervals $J_{n}=\left[\alpha_{n}, \beta_{n}\right]$ with lengths $\beta_{n}-\alpha_{n}=$ $n^{-1}$, such that

$$
\left|\mu_{1}\right|\left(J_{n}\right)+\left|\mu_{2}\right|\left(J_{n}\right)>2 b
$$

for every $n \geq 1$. By taking a subsequence we can assume the convergence $\alpha_{n} \rightarrow \bar{x} \in I$. This implies

$$
\left|\mu_{1}\right|(\{\bar{x}\})+\left|\mu_{2}\right|(\{\bar{x}\}) \geq 2 b .
$$

As defined in Section 2.5, the left hand side of (6.9) is the total strength of the two waves in the solution of the Riemann problem with left and right states $(v, u)(\bar{x}-),(v, u)(\bar{x}+)$. Since this solution takes values in the domain $\mathcal{D}_{a b}$, the sum of these two strengths must be $\leq 2(b-a)$. We thus reach a contradiction with (6.9), proving the second part of the lemma.

\section{$7 \quad$ Wave decay estimates}

In this section we prove two estimates on the decay of rarefaction waves, extending the analysis in Section 3 to general BV solutions.

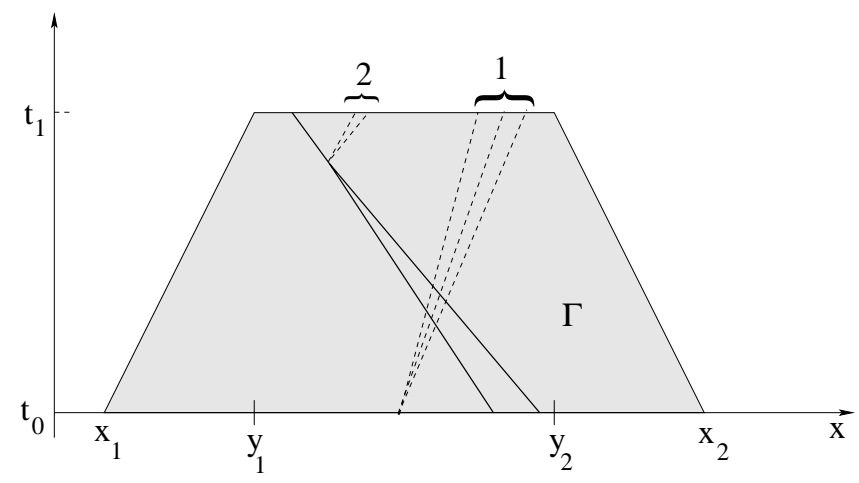

Figure 12: The two types of rarefaction waves which can cross the upper boundary of the trapezoidal domain $\Gamma$ at (7.2).

\subsection{Solutions without large shocks.}

We first study the simpler case where no large shock is present. Consider a BV solution of (1.1). Fix a time step $\Delta t$ and a space step $\Delta x$, such that

$$
\Delta x=2 \hat{\lambda} \cdot \Delta t,
$$

and consider a domain of the form (see Fig. 12)

$$
\Gamma=\left\{(t, x) ; \quad t \in\left[t_{0}, t_{1}\right], \quad x \in\left[x_{1}+\hat{\lambda}\left(t-t_{0}\right), x_{2}-\hat{\lambda}\left(t-t_{0}\right)\right]\right\},
$$


with

$$
t_{1}-t_{0}=\Delta t, \quad x_{2}-x_{1}=2 \Delta x .
$$

We seek an estimate on the total amount of rarefaction waves at time $t_{1}$, along the upper boundary

$$
\left[y_{1}, y_{2}\right]=\left[x_{1}+\hat{\lambda}\left(t_{1}-t_{0}\right), x_{2}-\hat{\lambda}\left(t_{1}-t_{0}\right)\right] .
$$

Notice that, by (7.1) and (7.3), this interval has length $y_{2}-y_{1}=\Delta x$.

As shown in Fig. 12, these rarefactions can be of two types:

1) Old rarefactions which were already present along the basis $\left[x_{1}, x_{2}\right]$ at time $t_{0}$. The total amount of these waves can be controlled because, as discussed in Section 3, their density has decayed during the entire time interval $\left[t_{0}, t_{1}\right]$. Roughly speaking, we have

$$
\begin{aligned}
& \text { [total amount of old rarefactions }] \leq\left(y_{2}-y_{1}\right) \cdot[\text { maximum density }] \\
& \quad \leq\left(y_{2}-y_{1}\right) \cdot \frac{\mathcal{O}(1)}{t_{1}-t_{0}}=\mathcal{O}(1) \cdot 2 \hat{\lambda}
\end{aligned}
$$

2) New rarefactions produced by wave interactions inside the domain $\Gamma$. Assuming that the total strength of all waves at the initial time $t_{0}$ is $\leq K_{0}$ and all shocks in $\Gamma$ have size $\leq \delta_{0}$, the total strength of these new waves will be of order $\mathcal{O}(1) \cdot \delta_{0} K_{0}^{2}$.

Lemma 7.1 Let $\mathcal{D}_{a b}$ be the domain in (6.2). Then one can find a constant $K_{a b}$ such that, for any given $K_{0}$, there exists $\delta_{0}>0$ for which the following holds.

Let $(v, u)$ be a $B V$ solution of (1.1) taking values inside $\mathcal{D}_{a b}$ and let $\Gamma$ be the trapezoid defined at 7.2). Assume that

(i) at time $t_{0}$ the total strength of all waves contained inside the lower boundary $\left[x_{1}, x_{2}\right]$ is $\leq K_{0}$, and

(ii) all shocks inside $\Gamma$ have strength $\leq \delta_{0}$.

Call $\hat{\mu}_{i}$ the measures of $i$-waves in the solutions at time $t_{1}$. Then the total strength of all rarefactions waves contained inside the upper boundary $\left[y_{1}, y_{2}\right]$ satisfies

$$
\left(\hat{\mu}_{1}^{+}+\hat{\mu}_{2}^{+}\right)\left(\left[y_{1}, y_{2}\right]\right) \leq K_{a b} .
$$

Notice that here the constant $K_{a b}$ can be large, but is independent of $K_{0}$. This implies that, if the initial data contain a large amount of waves but the solution does not develop large shocks, then most of the rarefaction waves present at time $t_{0}$ will disappear during the time interval $\left[t_{0}, t_{1}\right]$, being canceled by waves of the same family but opposite sign. Compared with the decay estimate proved in Chapter 10 of [4, the main difference is that here the total strength of waves can be large. However, thanks to Proposition 2.1, the total strength of new waves produced by interactions can be made arbitrarily small by choosing $\delta_{0}>0$ small enough. 
Proof. 1. Consider any interval $I \doteq[a, b] \subset\left[y_{1}, y_{2}\right]$. Call $t \mapsto \xi_{1}(t), t \mapsto \xi_{2}(t)$ respectively the minimal backward 2-characteristics passing through $a, b$ at time $t_{1}$. Motivated by (3.8), we define

$$
Z(t) \doteq \int_{\xi_{1}(t)}^{\xi_{2}(t)} \varphi(t, x) d x
$$

where

$$
\varphi(t, x)=\varphi\left(w_{2}(t, x)-w_{1}(t, x)\right)=\varphi(2 h(t, x))
$$

is the function defined at (3.4). As long as the solution $(v, u)$ takes values in $\mathcal{D}_{a b}$ we have $h(v) \geq a$, hence $\varphi$ remains bounded and uniformly positive. The integral (7.5) thus provides an equivalent way to measure the distance $\xi_{2}(t)-\xi_{1}(t)$ between the two characteristics.

Since the total variation is bounded and all characteristic speeds are bounded by $\hat{\lambda}$, the function $t \mapsto Z(t)$ is Lipschitz continuous. Its time derivative

$$
\dot{Z}=\dot{\xi}_{2} \cdot \varphi\left(\xi_{2}\right)-\dot{\xi}_{1} \cdot \varphi\left(\xi_{1}\right)+\int_{\xi_{1}}^{\xi_{2}} D_{t} \varphi
$$

is well defined for a.e. time $t$. Notice that here $D_{t} \varphi$ is a bounded measure. Its atomic part is supported on the set of shocks.

In the following, for any given time $t$ we denote by $\mathcal{S}$ the set of all shocks contained inside the interval $[a(t), b(t)]$ and call $k_{\alpha} \in\{1,2\}$ the family of the shock located at $x_{\alpha}(t)$. Moreover, by $D_{x}^{c}$ be denote the continuous (i.e., non atomic) part of a distributional derivative w.r.t. $x$, Motivated by (3.8), denoting by $c(x)=c(v(t, x))$ the wave speed and using (2.5), (17.6), we compute

$$
\begin{aligned}
\dot{Z}= & c\left(\xi_{2}\right) \varphi\left(\xi_{2}\right)-c\left(\xi_{1}\right) \varphi\left(\xi_{1}\right)-\int_{\xi_{1}}^{\xi_{2}} \varphi^{\prime} c \cdot\left(D_{x}^{c} w_{1}+D_{x}^{c} w_{2}\right) \\
& -\sum_{\alpha \in \mathcal{S}} \dot{x}_{\alpha} \cdot\left[\varphi\left(x_{\alpha}+\right)-\varphi\left(x_{\alpha}-\right)\right] \\
= & \int_{\xi_{1}}^{\xi_{2}}\left(c^{\prime} \varphi+c \varphi^{\prime}\right) \cdot\left(D_{x}^{c} w_{2}-D_{x}^{c} w_{1}\right)+\sum_{\alpha \in \mathcal{S}}\left[c\left(x_{\alpha}+\right) \varphi\left(x_{\alpha}+\right)-c\left(x_{\alpha}-\right) \varphi\left(x_{\alpha}-\right)\right] \\
& -\int_{\xi_{1}}^{\xi_{2}} \varphi^{\prime} c \cdot\left(D_{x}^{c} w_{1}+D_{x}^{c} w_{2}\right)-\sum_{\alpha \in \mathcal{S}} \dot{x}_{\alpha} \cdot\left[\varphi\left(x_{\alpha}+\right)-\varphi\left(x_{\alpha}-\right)\right] .
\end{aligned}
$$

For each shock $\alpha \in \mathcal{S}$, two cases must be considered.

CASE 1: The shock at $x_{\alpha}$ belongs to the first family. By definition, its strength is

$$
\sigma_{\alpha}=w_{1}\left(x_{\alpha}-\right)-w_{1}\left(x_{\alpha}+\right) \in\left[0, \delta_{0}\right] .
$$

In this case, we have

$$
\begin{gathered}
\dot{x}_{\alpha}=-\frac{c\left(x_{\alpha}+\right)+c\left(x_{\alpha}-\right)}{2}+\mathcal{O}(1) \cdot \sigma_{\alpha}^{2}, \\
\left|w_{2}\left(x_{\alpha}+\right)-w_{2}\left(x_{\alpha}-\right)\right|=\mathcal{O}(1) \cdot \sigma_{\alpha}^{3}, \\
\varphi\left(x_{\alpha}+\right)-\varphi\left(x_{\alpha}-\right)=\varphi^{\prime}\left(x_{\alpha}-\right) \sigma_{\alpha}+\mathcal{O}(1) \cdot \sigma_{\alpha}^{2}, \\
c\left(x_{\alpha}+\right)-c\left(x_{\alpha}-\right)=c^{\prime}\left(x_{\alpha}-\right) \sigma_{\alpha}+\mathcal{O}(1) \cdot \sigma_{\alpha}^{2},
\end{gathered}
$$


Using the fundamental relation $c^{\prime} \varphi+2 c \varphi^{\prime}=0$ we thus obtain

$$
\begin{aligned}
& {\left[c\left(x_{\alpha}+\right) \varphi\left(x_{\alpha}+\right)-c\left(x_{\alpha}-\right) \varphi\left(x_{\alpha}-\right)\right]-\dot{x}_{\alpha} \cdot\left[\varphi\left(x_{\alpha}+\right)-\varphi\left(x_{\alpha}-\right)\right]} \\
& \quad=\left[c^{\prime}\left(x_{\alpha}-\right) \varphi\left(x_{\alpha}-\right)+c\left(x_{\alpha}-\right) \varphi^{\prime}\left(x_{\alpha}-\right)\right] \sigma_{\alpha}+c\left(x_{\alpha}-\right) \varphi^{\prime}\left(x_{\alpha}-\right) \sigma_{\alpha}+\mathcal{O}(1) \cdot \sigma_{\alpha}^{2} \\
& \quad=\mathcal{O}(1) \cdot \sigma_{\alpha}^{2} .
\end{aligned}
$$

CASE 2: The shock at $x_{\alpha}$ belongs to the second family. By definition, its strength is

$$
\sigma_{\alpha}=w_{2}\left(x_{\alpha}-\right)-w_{2}\left(x_{\alpha}+\right) \in\left[0, \delta_{0}\right] .
$$

In this case, we have

$$
\begin{gathered}
\dot{x}_{\alpha}=\frac{c\left(x_{\alpha}+\right)+c\left(x_{\alpha}-\right)}{2}+\mathcal{O}(1) \cdot \sigma_{\alpha}^{2}, \\
\left|w_{1}\left(x_{\alpha}+\right)-w_{1}\left(x_{\alpha}-\right)\right|=\mathcal{O}(1) \cdot \sigma_{\alpha}^{3}, \\
\varphi\left(x_{\alpha}+\right)-\varphi\left(x_{\alpha}-\right)=-\varphi^{\prime}\left(x_{\alpha}-\right) \sigma_{\alpha}+\mathcal{O}(1) \cdot \sigma_{\alpha}^{2}, \\
c\left(x_{\alpha}+\right)-c\left(x_{\alpha}-\right)=-c^{\prime}\left(x_{\alpha}-\right) \sigma_{\alpha}+\mathcal{O}(1) \cdot \sigma_{\alpha}^{2},
\end{gathered}
$$

In this case we obtain

$$
\begin{aligned}
& {\left[c\left(x_{\alpha}+\right) \varphi\left(x_{\alpha}+\right)-c\left(x_{\alpha}-\right) \varphi\left(x_{\alpha}-\right)\right]-\dot{x}_{\alpha} \cdot\left[\varphi\left(x_{\alpha}+\right)-\varphi\left(x_{\alpha}-\right)\right]} \\
& \quad=\left[c^{\prime}\left(x_{\alpha}-\right) \varphi\left(x_{\alpha}-\right)+c\left(x_{\alpha}-\right) \varphi^{\prime}\left(x_{\alpha}-\right)\right] \sigma_{\alpha}-c\left(x_{\alpha}-\right) \varphi^{\prime}\left(x_{\alpha}-\right) \sigma_{\alpha}+\mathcal{O}(1) \cdot \sigma_{\alpha}^{2} \\
& \quad=c^{\prime}\left(x_{\alpha}-\right) \varphi\left(x_{\alpha}-\right) \sigma_{\alpha}+\mathcal{O}(1) \cdot \sigma_{\alpha}^{2} .
\end{aligned}
$$

From (7.7), using (7.8)-(7.9) and the relation $c^{\prime} \varphi+2 c \varphi^{\prime}=0$ one obtains

$$
\dot{Z}=\int_{\xi_{1}}^{\xi_{2}} c^{\prime} \varphi \cdot D_{x}^{c} w_{2}+\sum_{\alpha \in \mathcal{S}_{2}} c^{\prime}\left(x_{\alpha}-\right) \varphi\left(x_{\alpha}-\right) \sigma_{\alpha}+\mathcal{O}(1) \cdot \sum_{\alpha \in \mathcal{S}} \sigma_{\alpha}^{2} .
$$

Here the first summation ranges over the set $\mathcal{S}_{2}$ of all shocks of the second family, while the second summation ranges over the set of all shocks (of both families).

2. Call $\hat{\mu}_{2}$ the measure of 2 -waves in the solution at time $t_{1}$. For any $\varepsilon>0$ we can find finitely many intervals $\left[a_{\ell}, b_{\ell}\right], \ell=1, \ldots, m$, whose union contains nearly all positive 2 -waves, and very few negative 2 -waves. More precisely:

$$
\hat{\mu}_{2}^{+}\left(\left[y_{1}, y_{2}\right] \backslash \bigcup_{\ell}\left[a_{\ell}, b_{\ell}\right]\right) \leq \varepsilon, \quad \hat{\mu}_{2}^{-}\left(\bigcup_{\ell}\left[a_{\ell}, b_{\ell}\right]\right) \leq \varepsilon
$$

For each such interval, let $\xi_{\ell}(t), \tilde{\xi}_{\ell}(t)$ be the minimal backward 2-characteristics through $a_{\ell}, b_{\ell}$, respectively. Setting

$$
Z_{\ell}(t) \doteq \int_{\xi_{\ell}(t)}^{\tilde{\xi}_{\ell}(t)} \varphi(t, x) d x
$$

and applying (7.10) to each subinterval $\left[\xi_{\ell}, \tilde{\xi}_{\ell}\right]$ we obtain

$$
\sum_{\ell} \dot{Z}_{\ell}(t)=\int_{\xi_{\ell}}^{\tilde{\xi}_{\ell}} c^{\prime} \varphi \cdot D_{x}^{c} w_{2}+\sum_{\alpha \in \mathcal{S}_{2}} c^{\prime}\left(x_{\alpha}-\right) \varphi\left(x_{\alpha}-\right) \sigma_{\alpha}+\mathcal{O}(1) \cdot \sum_{\alpha \in \mathcal{S}} \sigma_{\alpha}^{2},
$$


where now $S_{2}$ and $\mathcal{S}$ refer to the shocks contained in the union of the intervals $\left[\xi_{\ell}(t), \tilde{\xi}_{\ell}(t)\right]$. For convenience, we introduce the constants

$$
0<\kappa_{\min } \doteq \min c^{\prime} \varphi, \quad \kappa_{\max } \doteq \max c^{\prime} \varphi, \quad \varphi_{\max } \doteq \max \varphi
$$

defined by taking the minimum and the maximum values of the functions $c^{\prime} \varphi$ and $\varphi$ over the domain $\mathcal{D}_{a b}$. Using Lemmas 2.3 and 2.4, the amounts of positive and negative 2-waves contained in the union of the intervals $\left[\xi_{\ell}(t), \tilde{\xi}_{\ell}(t)\right]$ at any time $t \in\left[t_{0}, t_{1}\right]$ can be estimated as

$$
\begin{aligned}
& \mu_{2}^{+}\left(\bigcup_{\ell}\left[\xi_{\ell}(t), \tilde{\xi}_{\ell}(t)\right]\right) \geq \mu_{2}^{+}\left(\bigcup_{\ell}\left[\xi_{\ell}\left(t_{1}\right), \tilde{\xi}_{\ell}\left(t_{1}\right)\right]\right)-\mathcal{O}(1) \cdot \delta_{0} \mu^{i n t}(\Gamma), \\
& \mu_{2}^{-}\left(\bigcup_{\ell}\left[\xi_{\ell}(t), \tilde{\xi}_{\ell}(t)\right]\right) \leq \mu_{2}^{-}\left(\bigcup_{\ell}\left[\xi_{\ell}\left(t_{1}\right), \tilde{\xi}_{\ell}\left(t_{1}\right)\right]\right)+\mathcal{O}(1) \cdot \delta_{0} \mu^{i n t}(\Gamma) .
\end{aligned}
$$

Combining (7.12) with (7.13)-(7.14) we obtain

$$
\begin{aligned}
\sum_{\ell} \dot{Z}_{\ell} & \geq \kappa_{\text {min }} \cdot \mu_{2}^{+}\left(\bigcup_{\ell}\left[\xi_{\ell}(t), \tilde{\xi}_{\ell}(t)\right]\right)-\kappa_{\max } \cdot \mu_{2}^{-}\left(\bigcup_{\ell}\left[\xi_{\ell}(t), \tilde{\xi}_{\ell}(t)\right]\right)+\mathcal{O}(1) \cdot \sum_{\alpha \in \mathcal{S}} \sigma_{\alpha}^{2} \\
& \geq \kappa_{\min } \cdot \hat{\mu}_{2}^{+}\left(\bigcup_{\ell}\left[a_{\ell}, b_{\ell}\right]\right)-\kappa_{\max } \cdot \hat{\mu}_{2}^{-}\left(\bigcup_{\ell}\left[a_{\ell}, b_{\ell}\right]\right)-\mathcal{O}(1) \cdot \delta_{0} \mu^{i n t}(\Gamma)-\mathcal{O}(1) \cdot \delta_{0} V(t) \\
& \geq \kappa_{\min } \cdot\left(\hat{\mu}_{2}^{+}\left(\left[y_{1}, y_{2}\right]\right)-\varepsilon\right)-\kappa_{\max } \cdot \varepsilon-\mathcal{O}(1) \cdot \delta_{0} \mu^{i n t}(\Gamma)-\mathcal{O}(1) \cdot \delta_{0} K_{0} \\
& \geq \kappa_{\text {min }} \cdot \hat{\mu}_{2}^{+}\left(\left[y_{1}, y_{2}\right]\right)-\mathcal{O}(1) \cdot \delta_{0} K_{0}^{2}-\mathcal{O}(1) \cdot \varepsilon .
\end{aligned}
$$

Observing that

$$
\sum_{\ell} Z_{\ell}\left(t_{0}\right) \geq 0, \quad \quad \sum_{\ell} Z_{\ell}\left(t_{1}\right) \leq\left(y_{2}-y_{1}\right) \varphi_{\max }
$$

and integrating (17.15) over the time interval $\left[t_{0}, t_{1}\right]$, we obtain

$$
\left(t_{1}-t_{0}\right) \kappa_{\min } \cdot \hat{\mu}_{2}^{+}\left(\left[y_{1}, y_{2}\right]\right) \leq\left(y_{2}-y_{1}\right) \varphi_{\max }+\mathcal{O}(1) \cdot\left(t_{1}-t_{0}\right) \delta_{0} K_{0}^{2}+\mathcal{O}(1) \cdot\left(t_{1}-t_{0}\right) \varepsilon .
$$

3. Since $y_{2}-y_{1}=\hat{\lambda}\left(t_{1}-t_{0}\right)$ and $\varepsilon>0$ can be taken arbitrarily small, (7.16) yields an a priori bound on the total amount of positive 2 -waves at the terminal time $t_{1}$, namely

$$
\hat{\mu}_{2}^{+}\left(\left[y_{1}, y_{2}\right]\right) \leq \hat{\lambda} \frac{\varphi_{\max }}{\kappa_{\min }}+C_{1} \delta_{0} K_{0}^{2}
$$

for a suitable constant $C_{1}$. Of course, an entirely similar estimate is valid for rarefaction waves of the first family.

For any given $K_{0}$, we can now choose $\delta_{0}>0$ so that

$$
\hat{\mu}_{1}^{+}\left(\left[y_{1}, y_{2}\right]\right)+\hat{\mu}_{2}^{+}\left(\left[y_{1}, y_{2}\right]\right) \leq 2 \hat{\lambda} \frac{\varphi_{\max }}{\kappa_{\min }}+2 C_{1} \delta_{0} K_{0}^{2} \leq 3 \hat{\lambda} \frac{\varphi_{\max }}{\kappa_{\min }} \doteq K_{a b} .
$$

With the above definition of the constant $K_{a b}$, the conclusion of the Lemma is achieved. 


\subsection{Solutions with one large shock.}

Our next goal is to extend Lemma 7.1 to the case where one large shock is present.

To fix the ideas, let $x=\gamma(t)$ be the location of a 1-shock, in a solution $U=(v, u)$ of (1.1). For a BV solution, the local behavior near the shock is well understood. The shock speed $t \mapsto \dot{\gamma}(t)$ is a BV function with at most countably many jumps. These occur on a countable set $\mathcal{T}$ of times where another shock (or a centered compression) impinges on $\gamma$. The left and right limits of the solution across the shock

$$
U^{l}(t) \doteq \lim _{x \rightarrow \gamma(t)-} U(t, x), \quad U^{r}(t) \doteq \lim _{x \rightarrow \gamma(t)+} U(t, x)
$$

are well defined for all times $t \notin \mathcal{T}$. In terms of these limits one can define the measures $\mu_{i}^{l}$, $\mu_{i}^{r}$ of waves to the left and to the right of the shock as follows.

Call $\left(w_{1}^{l}(t), w_{2}^{l}(t)\right)$ the Riemann coordinates of the state $U^{l}(t)$. Moreover, denote by $\mu_{i}^{(t)}$ the measure of $i$-waves in the solution $u(t, \cdot)$. Then

- The continuous (i.e., non atomic) part of $\mu_{i}^{l}$ coincides with the continuous part of $-D_{t} w_{i}^{l}$.

- If $\tau \in \mathcal{T}$ is a time where $U^{l}$ has a jump, then

$$
\mu_{1}^{l}(\{\tau\}) \doteq \lim _{\varepsilon \rightarrow 0+} \mu_{1}^{(\tau-\varepsilon)}([\gamma(\tau), \gamma(\tau-\varepsilon)[)
$$

is the amount of 1 -waves hitting the shock from the left, at time $\tau$. Moreover,

$$
\mu_{2}^{l}(\{\tau\}) \doteq \lim _{\varepsilon \rightarrow 0+} \mu_{2}^{(\tau-\varepsilon)}([\gamma(\tau)-\hat{\lambda} \varepsilon, \gamma(\tau)]) .
$$

is the amount of 2 -waves hitting the shock from the left, at time $\tau$.

Similarly, let $\left(w_{1}^{r}(t), w_{2}^{r}(t)\right)$ be the Riemann coordinates of the state $U^{r}(t)$. Then

- The continuous part of $\mu_{1}^{r}$ coincides with the continuous part of $D_{t} w_{1}^{r}$. The continuous part of $\mu_{2}^{r}$ coincides with the continuous part of $-D_{t} w_{2}^{r}$.

- If $\tau \in \mathcal{T}$ is a time where $U^{r}$ has a jump, then

$$
\left.\left.\mu_{1}^{r}(\{\tau\}) \doteq \lim _{\varepsilon \rightarrow 0+} \mu_{1}^{(\tau-\varepsilon)}(] \gamma(\tau-\varepsilon), \gamma(\tau)+\hat{\lambda} \varepsilon\right]\right)
$$

is the amount of 1-waves hitting the shock from the right, at time $\tau$, while

$$
\mu_{2}^{r}(\{\tau\}) \doteq \lim _{\varepsilon \rightarrow 0+} \mu_{2}^{(\tau+\varepsilon)}([\gamma(\tau), \gamma(\tau)+\hat{\lambda} \varepsilon])
$$

is the amount of 2-waves coming out from the shock from the right.

For an analysis of the local structure of a solution in the neighborhood of a point, we refer to [9, 15] 

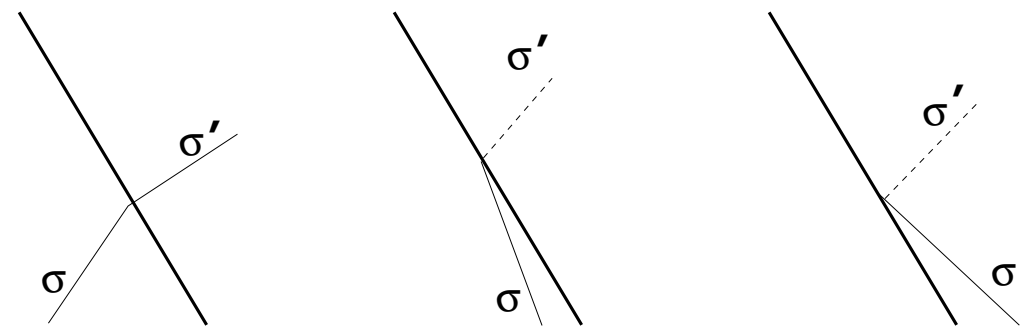

Figure 13: As long as all states remain inside the domain $\mathcal{D}_{a b}$, the strength of the 2-wave emerging from the interaction of a small wave with a large 1-shock satisfies the bounds in Lemma 2.1 .

Recalling (7.1), we now consider the trapezoidal domain $\widetilde{\Gamma}$ shown in Fig. 14 where the left side is a large 1-shock. More precisely:

$$
\left.\left.\widetilde{\Gamma}=\left\{(t, x) ; t \in\left[t_{0}, t_{1}\right], \quad x \in\right] \gamma(t), x_{2}-\hat{\lambda}\left(t-t_{0}\right)\right]\right\}
$$

where now

$$
t_{1}-t_{0}=\Delta t, \quad x_{2}-\hat{\lambda}\left(t_{1}-t_{0}\right)-\gamma\left(t_{1}\right) \leq 2 \Delta x
$$

In order to estimate the strength of 2-waves emerging from interactions with the large 1-shock, we rely on the following elementary estimate.

By taking limits of front tracking approximations and using the lower semicontinuity of wave measures w.r.t. $\mathbf{L}^{1}$ convergence (proved in [4]), from Lemma 2.1 one obtains

Lemma 7.2 Consider a 1-shock, located along the curve $\left\{x=\gamma(t) ; t \in\left[t_{0}, t_{1}\right]\right\}$. For $i=1,2$, call $\mu_{i}^{l}, \mu_{i}^{r}$ the measures of $i$-waves to the left and to the right of the shock, defined as above. Then the total strength of 2-waves emerging from the shock is estimated by

$$
\left|\mu_{2}^{r}\right|\left(\left[t_{0}, t_{1}\right]\right) \leq C_{\gamma} \cdot\left(\left|\mu_{1}^{l}\right|+\left|\mu_{2}^{l}\right|\right)\left(\left[t_{0}, t_{1}\right]\right)+\left|\mu_{1}^{r}\right|\left(\left[t_{0}, t_{1}\right]\right) .
$$

Because of the above estimates, it is convenient to measure the weighted strength of all small waves contained in $\Gamma$ at time $t$ by assigning a larger weight to waves which are approaching the large 1-shock. Namely:

$$
\left.\left.W(t) \doteq\left(2\left|\mu_{1}\right|+\left|\mu_{2}\right|\right)(] \gamma(t), x_{2}-\hat{\lambda}\left(t-t_{0}\right)\right]\right) .
$$

As before, we seek an estimate on the total strength of rarefaction waves at time $t_{1}$. As shown in Fig [14, three different types of rarefactions can now occur.

1) Old rarefactions, which were already present along the bottom side $\left[\gamma(t), x_{2}\right]$ at time $t_{0}$. Because of genuine nonlinearity, these waves decay. Their total strength at time $t_{1}$ is uniformly bounded, regardless of the amount of waves at the initial time $t_{0}$.

2) New rarefactions, produced by interactions of small waves inside the domain $\widetilde{\Gamma}$. By the interaction estimates (2.14)-(2.16), the total strength of these waves can be rendered arbitrarily small, by choosing $\delta_{0}$ small enough. 
3) 2-rarefactions emerging from the large shock. These occur when (i) a 2-rarefaction crosses the large shock, or (ii) a 1-shock (or a 1-compression wave) hits the large 1-shock from the left or from the right. Because of the definition (17.23), the total strength of these waves is controlled by the decay in the functional $W(\cdot)$.

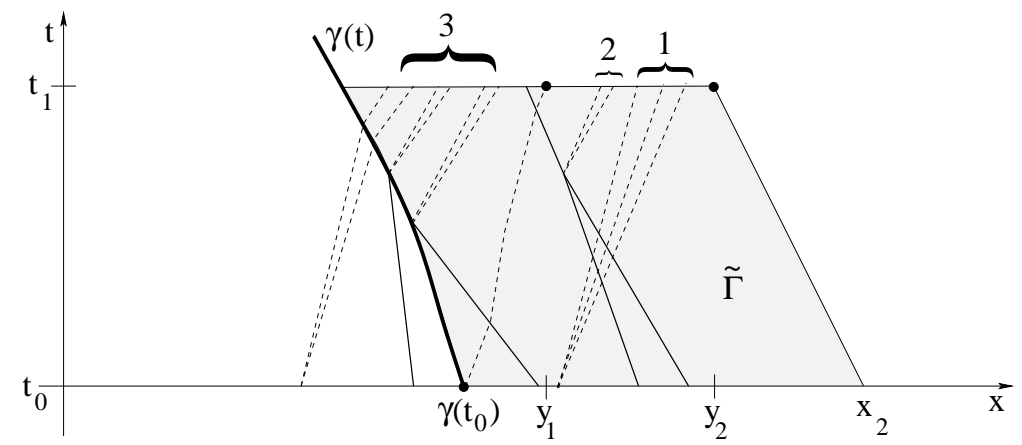

Figure 14: The three types of rarefaction waves which can cross the upper boundary of the large trapezoidal domain $\Gamma$ at (7.20). Here $y_{1}$ is the largest point such that the minimal backward 2characteristic through $\left(t_{1} . y_{1}\right)$ meets the 1 -shock $\gamma$.

Lemma 7.3 Consider the domain $\mathcal{D}_{a b}$ in (6.2). Then one can find a constant $\widetilde{K}_{a b}$ such that, for any given $\widetilde{K}_{0}$, there exists $\delta_{0}>0$ for which the following holds.

Let $(v, u)$ be a $B V$ solution of (1.1) taking values inside $\mathcal{D}_{a b}$ and let $\widetilde{\Gamma}$ be the domain in (7.20)-17.21), shown in Fig. 14. Assume that:

(i) At time $t_{0}$ the total weighted strength of all waves along the lower boundary $\left[\gamma\left(t_{0}\right), x_{2}\right]$ is $W\left(t_{0}\right) \leq \widetilde{K}_{0}$.

(ii) The total strength of all waves impinging on the large 1-shock from the left is $\leq 3 b$.

(iii) All shocks inside $\widetilde{\Gamma}$ have strength $\leq \delta_{0}$.

Then, calling $\hat{\mu}_{1}, \hat{\mu}_{2}$ the wave measures at time $t_{1}$, the total strength of all rarefactions contained inside the upper boundary of $\widetilde{\Gamma}$ satisfies

$$
\left(\hat{\mu}_{1}^{+}+\hat{\mu}_{2}^{+}\right)\left(\left[\gamma\left(t_{1}\right), x_{2}-\hat{\lambda}\left(t_{1}-t_{0}\right)\right]\right) \leq \widetilde{K}_{a b}+\left[W\left(t_{0}\right)-W\left(t_{1}\right)\right] .
$$

Proof. 1. As shown in Fig. 14, consider the points

$y_{1} \doteq \inf \left\{y>\gamma\left(t_{1}\right) ;\right.$ the minimal 2-characteristic $\xi$ through $\left(t_{1}, y\right)$ satisfies $\left.\xi\left(t_{0}\right) \geq \gamma\left(t_{0}\right)\right\}$.

$$
y_{2} \doteq x_{2}-\hat{\lambda}\left(t_{1}-t_{0}\right)
$$

From (7.21) we deduce

$$
y_{2}-\gamma\left(t_{1}\right) \leq 2 \Delta x, \quad x_{2}-\gamma\left(t_{0}\right) \leq 3 \Delta x .
$$

We observe that, at time $t_{1}$, all 1-rarefactions located inside the interval $\left[\gamma\left(t_{1}\right), y_{2}\right]$ are of Type 1 (old rarefactions already present at time $t_{0}$ or Type 2 (new rarefactions generated by 
wave interactions inside $\widetilde{\Gamma}$ ). These can be estimated by the same techniques as in Lemma 7.1 . Similarly, at time $t_{1}$ all 2-rarefactions located inside the interval $\left[y_{1}, y_{2}\right]$ are of Type 1 or 2 , and can be bounded in the same way. Calling $\hat{\mu}_{i}$ the measure of $i$-waves at time $t_{1}$, by choosing $\delta_{0}>0$ sufficiently small we can thus conclude

$$
\hat{\mu}_{1}^{+}\left(\left[\gamma\left(t_{1}\right), y_{2}\right]\right) \leq K_{a b}, \quad \hat{\mu}_{2}^{+}\left(\left[y_{1}, y_{2}\right]\right) \leq K_{a b} .
$$

for a suitable constant $K_{a b}$.

2. To prove the lemma, it thus remains to bound the total amount of 2-rarefactions contained in the interval $\left[\gamma\left(t_{1}\right), y_{1}[\right.$. This can be achieved by standard interaction estimates. Indeed, using (7.22) we obtain

$$
\begin{aligned}
& \left|\hat{\mu}_{2}\right|\left(\left[\gamma\left(t_{1}\right), y_{2} \mid\right) \leq \text { total strength of } 2\right. \text {-waves emerging from the big 1-shock] } \\
& \quad+\text { total strength of new waves generated by interactions within } \widetilde{\Gamma}] \\
& \quad \leq\left|\mu_{2}^{r}\right|\left(\left[t_{0}, t_{1}\right]\right)+\mathcal{O}(1) \cdot \delta_{0} \\
& \left.\left.\left.\left.\quad \leq C_{\gamma} \cdot\left(\left|\mu_{1}^{l}\right|+\left|\mu_{2}^{l}\right|\right)(] t_{0}, t_{1}\right]\right)+\left|\mu_{1}^{r}\right|(] t_{0}, t_{1}\right]\right)+\mathcal{O}(1) \cdot \delta_{0} .
\end{aligned}
$$

The assumption (ii) implies

$$
C_{\gamma} \cdot\left(\left|\mu_{1}^{l}\right|+\left|\mu_{2}^{l}\right|\right)\left(\left[t_{0}, t_{1}\right]\right) \leq 3 b C_{\gamma} .
$$

By Lemma 2.1, when a 1-wave impinges on the large 1-shock from the right, the outgoing 2wave resulting from the interaction has smaller strength. By our definition of $W$, the incoming 1 -wave has weight 2 while the outgoing 2 -wave has weight 1 . Keeping this in mind, we obtain $W\left(t_{1}\right)-W\left(t_{0}\right) \leq C_{\gamma} \cdot[$ total strength of waves impinging on the big 1-shock from the left] - [total strength of 1-waves impinging on the big 1-shock from the right] $+\mathcal{O}(1) \cdot[$ total strength of new waves generated by interactions within $\widetilde{\Gamma}]$

$$
\left.\left.\left.\left.\leq C_{\gamma} \cdot\left(\left|\mu_{1}^{l}\right|+\left|\mu_{2}^{l}\right|\right)(] t_{0}, t_{1}\right]\right)-C_{\gamma} \cdot\left|\mu_{1}^{r}\right|(] t_{0}, t_{1}\right]\right)+\mathcal{O}(1) \cdot \delta_{0}
$$

Combining (7.29) with (7.28) we obtain

$$
\left.\left.\left|\mu_{1}^{r}\right|(] t_{0}, t_{1}\right]\right) \leq W\left(t_{0}\right)-W\left(t_{1}\right)+3 b C_{\gamma}+\mathcal{O}(1) \cdot \delta_{0} .
$$

Inserting the bound (7.30) in (7.27) we finally obtain

$$
\left|\hat{\mu}_{2}\right|\left(\left[\gamma\left(t_{1}\right), y_{2}[) \leq 3 b C_{\gamma}+W\left(t_{0}\right)-W\left(t_{1}\right)+3 b C_{\gamma}+\widetilde{C} \delta_{0}\right.\right.
$$

where $\widetilde{C}$ is a constant depending only on $a, b$, and $\widetilde{K}_{0}$. We can now choose $\delta_{0}>0$ small enough so that

$$
\left|\hat{\mu}_{2}\right|\left(\left[\gamma\left(t_{1}\right), y_{2}[) \leq 7 b C_{\gamma}+W\left(t_{0}\right)-W\left(t_{1}\right)\right.\right.
$$

Defining

$$
\widetilde{K}_{a b} \doteq 2 K_{a b}+7 b C_{\gamma},
$$

in view of (17.26) and (7.32) we achieve the desired bound (7.24). 
Remark 7.1 The estimate (17.4) remains valid if the domain $\Gamma$ in (17.2) is replaced by

$$
\Gamma^{\prime}=\left\{(t, x) ; t \in\left[t_{0}, t_{1}\right], \quad \max \left\{x_{1}+\hat{\lambda}, \gamma(t)\right\} \leq x \leq \min \left\{x_{2}-\hat{\lambda}\left(t-t_{0}\right), \tilde{\gamma}(t)\right\}\right\},
$$

where $\gamma(t)$ is the location of a 2-shock, while $\tilde{\gamma}(t)$ is the location of a 1-shock (see Fig. 15, left). Indeed, no wave of any kind can enter $\Gamma^{\prime}$ from the left boundary $\gamma$, nor from the right boundary $\tilde{\gamma}$.

Similarly, assume that the domain $\widetilde{\Gamma}$ in (17.20) is replaced by

$$
\widetilde{\Gamma}^{\prime}=\left\{(t, x) ; \quad t \in\left[t_{0}, t_{1}\right], \quad \gamma(t)<x<\min \left\{x_{2}-\hat{\lambda}\left(t-t_{0}\right), \tilde{\gamma}(t)\right\}\right\},
$$

where $\tilde{\gamma}(t)$ is the location of a 1-shock (see Fig. 15, right). Then, setting

$$
\left.y_{1} \doteq \gamma\left(t_{1}\right), \quad y_{2} \doteq \min x_{2}-\hat{\lambda}\left(t_{1}-t_{0}\right), \tilde{\gamma}\left(t_{1}\right)\right\}
$$

the same arguments used in the proof of Lemma 7.3 yield

$$
\left(\hat{\mu}_{1}^{+}+\hat{\mu}_{2}^{+}\right)\left(\left[y_{1}, y_{2}\right]\right) \leq \widetilde{K}_{a b}+\left[W\left(t_{0}\right)-W\left(t_{1}\right)\right] .
$$

Here $W(t)$ denotes the total weighted strength of waves at time $t$ contained inside $\widetilde{\Gamma}^{\prime}$. Indeed, no wave of any kind can enter $\widetilde{\Gamma}^{\prime}$ from the right boundary $\tilde{\gamma}$.
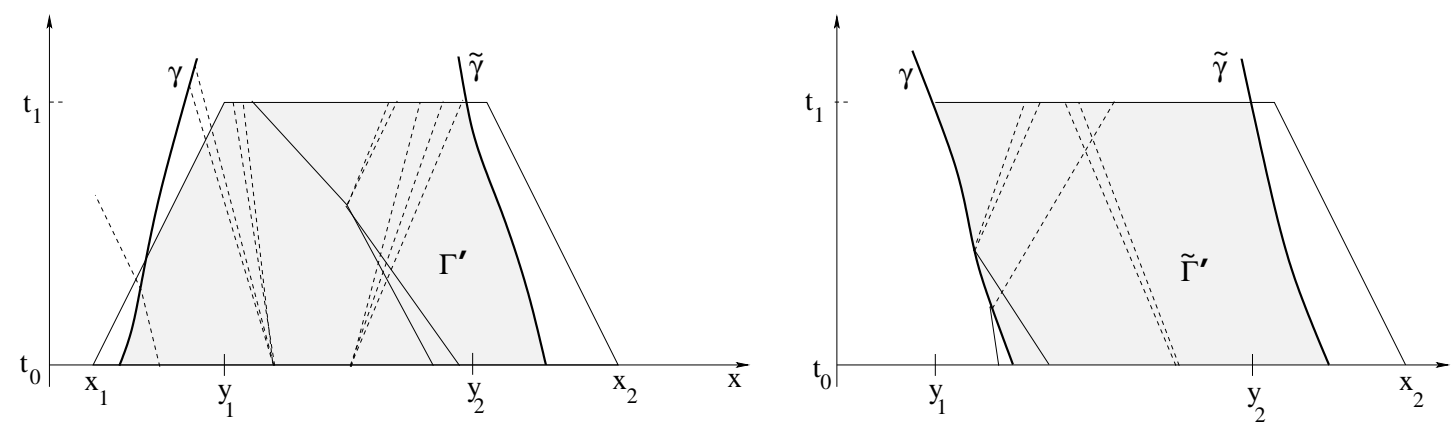

Figure 15: The domains $\Gamma^{\prime}$ and $\widetilde{\Gamma}^{\prime}$ considered in Remark 7.1

\section{Proof of the main theorem}

Toward a proof of Theorem 6.1, consider a solution $U=(v, u)$ of (1.1), taking values inside the domain $\mathcal{D}_{a b}$, and assume that

$$
\limsup _{t \rightarrow T-} \text { Tot.Var. }\{U(t, \cdot)\}=+\infty \text {. }
$$

As remarked in (6.4), blow up of the total variation can occur only in the region where $|x| \leq R_{0}+\hat{\lambda} T$. By a compactness argument, there exists a point $\bar{x}$ such that, for every $\varepsilon>0$,

$$
\limsup _{t \rightarrow T-} \text { Tot.Var. }\{U(t, \cdot) ;[\bar{x}-\varepsilon, \bar{x}+\varepsilon]\}=+\infty
$$


If the conclusion of Theorem 6.1 is violated, then there exists $\varepsilon>0$ such that the rectangular region

$$
\{(t, x) ; \quad t \in[T-\varepsilon, T], \quad|x-\bar{x}| \leq \varepsilon\}
$$

contains only finitely many shocks. By possibly shrinking the value of $\varepsilon$, we can assume that all these shocks meet at the point $\bar{P}=(T, \bar{x})$. In the next steps of the proof we will derive a contradiction, showing that the total variation of $U(t, \cdot)$ in a neighborhood of $\bar{P}$ remains uniformly bounded.

1. To fix the ideas, call $x=\gamma_{i}(t), t \in\left[t_{0}, T\right]$ the position of the $i$-th large shock. As shown in Fig. 16 we can assume that all these shocks reach the point $\bar{P}$ at time $t=T$, and moreover

$$
\gamma_{1}(t)<\cdots<\gamma_{m}(t)<\gamma_{m+1}(t)<\cdots<\gamma_{m+n}(t), \quad T-\varepsilon<t<T .
$$

Here $\gamma_{1}, \ldots, \gamma_{m}$ are 2-shocks while $\gamma_{m+1}, \ldots, \gamma_{m+n}$ are 1-shocks. Consider the domain

$$
\Gamma_{\varepsilon} \doteq\{(t, x) ; \quad t \in[T-\varepsilon, T], \quad \bar{x}-\varepsilon-\hat{\lambda}(T-t)<x<\bar{x}+\varepsilon+\hat{\lambda}(T-t)\} .
$$

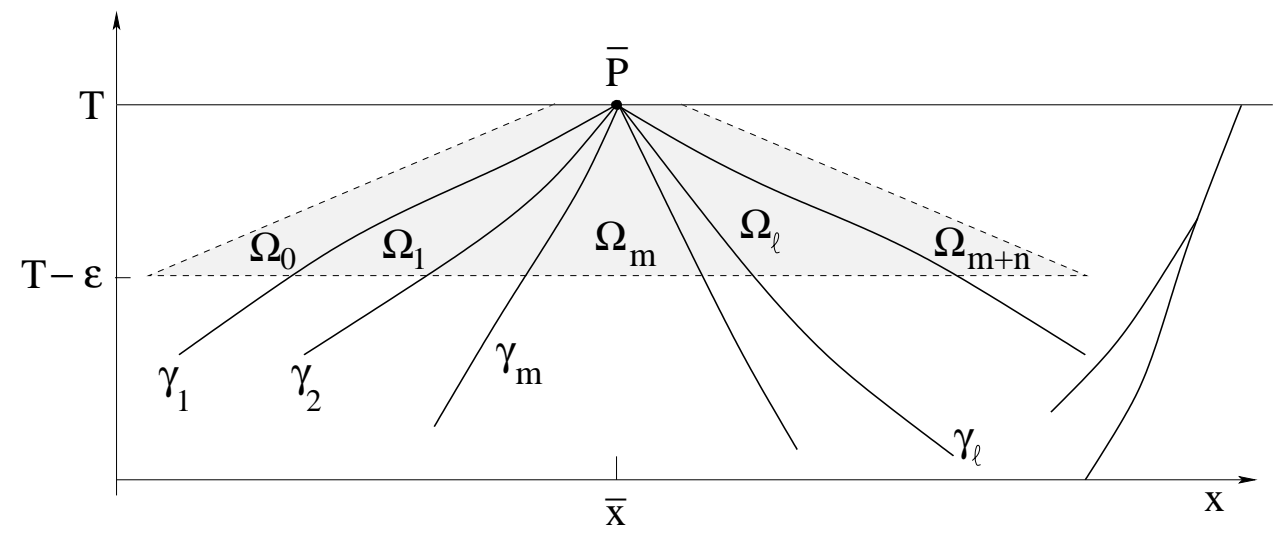

Figure 16: By taking a sufficiently small neighborhood of $\bar{P}=(T, \bar{x})$ one can assume that all shock curves $\gamma_{\ell}$ actually meet at $\bar{P}$. We illustrate here the case $m=n=3$. The shaded region is the set $\Gamma_{\varepsilon}$ in (8.4).

Set $t_{0} \doteq T-\varepsilon$ and let $\Omega_{0}, \Omega_{1}, \ldots, \Omega_{m+n}$ be the regions between these large shocks, so that

$$
\Omega_{\ell} \doteq\left\{(t, x) ; \quad \gamma_{\ell}(t)<x<\gamma_{\ell+1}(t), \quad t_{0}<t<T\right\} .
$$

For convenience, we denote by

$$
\gamma_{0}(t) \doteq \bar{x}-\varepsilon-\hat{\lambda}(T-t), \quad \gamma_{m+n+1}(t) \doteq \bar{x}+\varepsilon+\hat{\lambda}(T-t),
$$

the left and the right side of the trapezoid $\Gamma_{\varepsilon}$. By an inductive argument, we will show that the total variation of the solution restricted to each $\Omega_{\ell}$ is uniformly bounded.

2. To prove that the total variation on the middle domain $\Omega_{m}$ remains bounded, we choose mesh sizes $\Delta t, \Delta x$, with

$$
\Delta x=2 \hat{\lambda} \cdot \Delta t
$$


As shown in Fig. 17, left, we can cover the domain $\Omega_{m}$ with trapezoids whose basis has length $\leq 2 \Delta x$ and such that the bottom side of each trapezoid is the union of the top sides of the two lower ones.

More precisely, we set

$$
t_{j}=t_{0}+j \cdot \Delta t
$$

and cover $\Omega_{m}$ with trapezoids of the form (see Fig. 17, left)

$$
\begin{aligned}
\Gamma_{j k}=\Omega_{m} & \cap\left\{(t, x) ; t \in\left[t_{j}, t_{j+1}\right],\right. \\
& \left.k \cdot \Delta x+j \hat{\lambda} \cdot \Delta t+\left(t-t_{j}\right) \hat{\lambda}<x<(k+2) \cdot \Delta x+j \hat{\lambda} \cdot \Delta t-\left(t-t_{j}\right) \hat{\lambda}\right\} .
\end{aligned}
$$

Choosing the mesh $\Delta x>0$ small enough, by Lemma 6.1 we can assume that at time $t=t_{0}$ the total strength of waves along the bottom side of every trapezoid $\Gamma_{0, k}$ is $\leq 2 b$.

Let $K_{a b}$ be the constant in Lemma 7.1, depending only on $a, b$. We then choose

$$
K_{0}=2\left(b+2 K_{a b}\right)
$$

and find some $\delta_{0}>0$ so that the conclusion of Lemma 7.1 holds.

We claim that, if all shocks inside $\Omega_{m}$ have strength $\leq \delta_{0}$, then for every $j, k$ one has

$$
\text { [total strength of waves along the bottom side of } \left.\Gamma_{j, k}\right] \leq K_{0} \text {. }
$$

Indeed, since $K_{0}>2 b$, this is trivially true when $j=0$.

Now assume that (8.8) holds for some $j$ and all $k$. For a fixed $k$, consider the trapezoid $\Gamma_{j+1, k}$. Observe that the bottom side of $\Gamma_{j+1, k}$ is the union of the top sides of the two lower trapezoids $\Gamma_{j, k}$ and $\Gamma_{j, k+1}$. By the inductive assumption, an application of Lemma 4 implies that the total strength of rarefactions contained in the top side of $\Gamma_{j, k}$ is $\leq K_{a b}$, and the same is true for the top side of the trapezoid $\Gamma_{j, k+1}$. By (i) in Lemma 6.1 we conclude that the total strength of all waves contained in the bottom side of $\Gamma_{j+1, k}$ is $\leq 2\left(b+2 K_{a b}\right)=K_{0}$. Hence (8.8) holds also with $j$ replaced by $j+1$. By induction, the same estimate holds for every $j \geq 0$ and every $k$.

Since $\Omega_{m}$ can be covered with finitely many trapezoids $\Gamma_{j, k}$, this proves that the total variation of the solution remains uniformly bounded inside $\Omega_{m}$.

3. Next, assume that the total variation remains bounded on the domains $\Omega_{m}, \ldots, \Omega_{\ell-1}$. We claim that it also remain bounded on $\Omega_{\ell}$, the region to the right of the 1 -shock $\gamma_{\ell}$.

Toward this goal, we consider the trapezoids

$$
\begin{aligned}
\Gamma_{j k}=\Omega_{\ell} \cap & \left\{(t, x) ; t \in\left[t_{j}, t_{j+1}\right],\right. \\
& \left.k \cdot \Delta x+j \hat{\lambda} \cdot \Delta t+\left(t-t_{j}\right) \hat{\lambda}<x<(k+2) \cdot \Delta x+j \hat{\lambda} \cdot \Delta t-\left(t-t_{j}\right) \hat{\lambda}\right\} .
\end{aligned}
$$

but only for values of $j, k$ such that $\Gamma_{j, k}$ remains entirely to the right of the shock curve $\gamma_{\ell}$, namely

$$
\gamma_{\ell}\left(t_{j}\right)<k \cdot \Delta x+j \hat{\lambda} \cdot \Delta t
$$



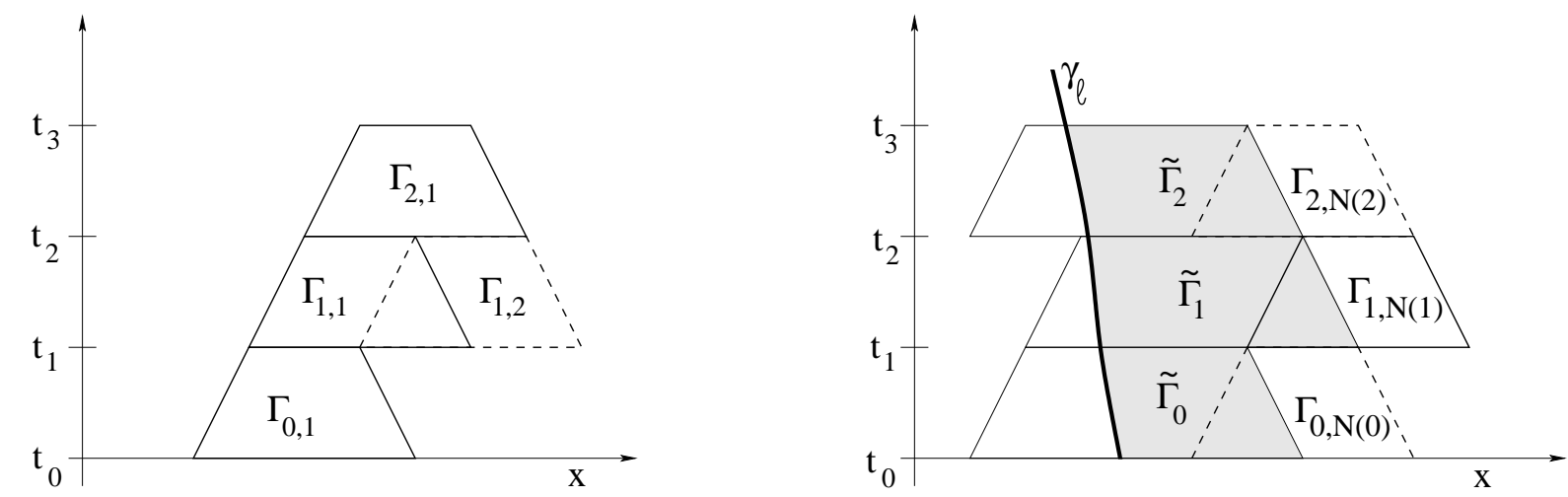

Figure 17: Left: Covering the domain $\Omega_{m}$ with trapezoids $\Gamma_{j, k}$. Notice that the bottom side of $\Gamma_{2,1}$ is the union of the top sides of the two lower trapezoids $\Gamma_{1,1}$ and $\Gamma_{1,2}$. Right: Covering the domains $\Omega_{\ell}$ with trapezoids. Here the B-trapezoids are shaded in grey. Notice that the bottom side of the boundary trapezoid $\widetilde{\Gamma}_{2}$ coincides with the top side of the $\widetilde{\Gamma}_{1}$, while the bottom side of $\widetilde{\Gamma}_{1}$ is the union of the top sides of $\widetilde{\Gamma}_{0}$ and $\Gamma_{0, N(0)}$. Similarly, the bottom side of $\Gamma_{2, N(2)}$ is contained in the union of the top sides of $\widetilde{\Gamma}_{1}$ and $\Gamma_{1, N(1)}$.

These will be called I-trapezoids. In addition, for each $j$ we consider a somewhat wider trapezoid, of the form

$$
\widetilde{\Gamma}_{j} \doteq\left\{(t, x) ; t \in\left[t_{j}, t_{j+1}\right], \quad \gamma_{\ell}(t)<x<(N(j)+1) \cdot \Delta x+j \hat{\lambda} \cdot \Delta t-\left(t-t_{j}\right) \hat{\lambda}\right\} .
$$

Here we choose $N(j)$ to be the smallest integer such that

$$
\gamma_{\ell}\left(t_{j+1}\right) \leq N(j) \cdot \Delta x+(j+1) \hat{\lambda} \cdot \Delta t .
$$

The $\widetilde{\Gamma}_{j}$ will be called B-trapezoids, since they touch the left boundary of $\Omega_{\ell}$. Since $\gamma_{\ell}$ is a 1-shock with speed $-\hat{\lambda}<\dot{\gamma}_{\ell}<0$, the above choice of $N(j)$ guarantees that the lengths of the top and the bottom side of $\widetilde{\Gamma}_{j}$ satisfy

$$
\left\{\begin{array}{l}
\Delta x \leq\left[\text { length of } \widetilde{\Gamma}_{j}^{t o p}\right] \leq 2 \Delta x, \\
\Delta x \leq\left[\text { length of } \widetilde{\Gamma}_{j}^{b o t t o m}\right] \leq 3 \Delta x .
\end{array}\right.
$$

We also observe that

- The bottom side of each I-trapezoid $\Gamma_{j, k}$ is contained in the union of the top sides of two I-trapezoids, or an I-trapezoid and a B-trapezoid.

- The bottom side of the B-trapezoid $\widetilde{\Gamma}_{j}$ is contained in the union of the top side of the lower B-trapezoid $\widetilde{\Gamma}_{j-1}$ and (possibly) the top side of some I-trapezoid.

By Lemma 6.1, (and the same arguments applied to the measures $\mu_{i}^{l}, i=1,2$ of waves impinging on the shock $\gamma_{\ell}$ from the left), we can choose the mesh sizes $\Delta t, \Delta x$ in (8.6) small enough so that

- The total strength of all waves on the bottom side of $\widetilde{\Gamma}_{0}$ and on the bottom side of each trapezoid $\Gamma_{0, k}$ is $\leq 2 b$. 
- During every time interval $\left[t_{j}, t_{j+1}\right]$, the total amount of waves impinging on $\gamma_{\ell}$ from the left is $\leq 2 b$.

4. Let $K_{a b}$ and $\widetilde{K}_{a b}$ be the constants for which the conclusions of Lemma 7.1 and of Lemma 7.3 hold. Of course, it is not restrictive to assume that $\widetilde{K}_{a b}>K_{a b}>1$. Choose

$$
K_{0} \doteq 2\left(b+\widetilde{K}_{a b}+4\left(b+K_{a b}\right)+K_{a b}\right)
$$

in Lemma 7.1 and

$$
\widetilde{K}_{0} \doteq 2 K_{0}+2\left(b+2 K_{a b}\right)
$$

in Lemma 7.3, and let $\delta_{0}>0$ be a constant small enough so that the conclusions of both lemmas hold.

With the above choices, we claim that, for every $j, k$,

(i) The total strength of all waves on the bottom side of each trapezoid $\Gamma_{j k}$ is $\leq K_{0}$.

(ii) The weighted strength of all waves on the bottom side of each trapezoid $\widetilde{\Gamma}_{j}$ is $W_{j}\left(t_{j}\right) \leq$ $2 \widetilde{K}_{0}$.

Indeed, by the choice of the step size $\Delta x$, both claims (i)-(ii) are true at time $t_{0}=T-\varepsilon$, i.e. for $j=0$ and any $k$.

Arguing by induction, assume that the above claims are true for the trapezoids $\Gamma_{j k}$ and $\widetilde{\Gamma}_{j}$, for a given $j$ and all $k$. We need to prove that they hold for the trapezoids $\Gamma_{j+1, k}$ and $\widetilde{\Gamma}_{j+1}$ as well.

Call $W_{j}(t)$ the total weighted strength of waves in the trapezoid $\widetilde{\Gamma}_{j}$ at time $t \in\left[t_{j}, t_{j}\right]$. By the inductive assumption $W_{j}\left(t_{j}\right) \leq 2 \widetilde{K}_{a b}$. Moreover, by our choice of $\Delta t$ the total strength of waves impinging on the 1 -shock $\gamma_{\ell}$ (the left boundary of $\widetilde{\Gamma}_{j}$ ) is $\leq 2 b$. We can thus apply Lemma 7.3 and conclude

[total strength of all rarefactions on the upper boundary of $\left.\widetilde{\Gamma}_{j}\right] \leq \widetilde{K}_{a b}+\left[W_{j}\left(t_{j}\right)-W_{j}\left(t_{j+1}\right)\right]$.

Two cases must be considered.

CASE 1: $W_{j}\left(t_{j}\right)-W_{j}\left(t_{j+1}\right) \geq 4\left(b+K_{a b}\right)$.

Consider the bottom side of $\widetilde{\Gamma}_{j+1}$. As shown in Fig. 17 left, this is contained in the union of the top side of $\widetilde{\Gamma}_{j}$ and (possibly) some smaller trapezoid $\Gamma_{j, N(j)}$. By Lemma 7.1 and part (i) of Lemma 6.1, the total strength of waves on the upper boundary of $\Gamma_{j, N(j)}$ is $\leq 2\left(b+K_{a b}\right)$.

Hence the total weighted strength of all waves on the lower boundary of $\widetilde{\Gamma}_{j+1}$ satisfies the bound

$$
W_{j+1}\left(t_{j+1}\right) \leq W_{j}\left(t_{j+1}\right)+2 \cdot 2\left(b+K_{a b}\right) \leq W_{j}\left(t_{j}\right) \leq 2 \widetilde{K}_{0} .
$$

CASE $2: W_{j}\left(t_{j}\right)-W_{j}\left(t_{j+1}\right)<4\left(b+K_{a b}\right)$. 
In this case, by Lemma 7.3 the total strength of waves on the upper boundary of $\widetilde{\Gamma}_{j}$ is $<\widetilde{K}_{a b}+4\left(b+K_{a b}\right)$. Since the total strength of positive waves on the upper boundary of the small trapezoid $\Gamma_{j, N(j)}$ is $\leq K_{a b}$, using again Lemma 6.1 we conclude that the total weighted strength of all waves on the lower boundary of $\widetilde{\Gamma}_{j+1}$ satisfies

$$
W_{j+1}\left(t_{j+1}\right) \leq 4\left(b+\left(\widetilde{K}_{a b}+4\left(b+K_{a b}\right)\right)+K_{a b}\right) \leq 2 \widetilde{K}_{0} .
$$

Together, 8.13) and (8.14) yield (ii), with $j$ replaced by $j+1$.

To prove (i), we again consider two cases.

CASE 1: the lower boundary of $\Gamma_{j+1, k}$ is contained in the union of the upper boundaries of the two small trapezoids $\Gamma_{j, k}$ and $\Gamma_{j, k+1}$.

In this case, as in step 2, using Lemma 7.1 and Lemma 6.1 we obtain

$$
\text { [total strength of waves on the lower boundary of } \left.\Gamma_{j, k+1}\right] \leq 2\left(b+2 K_{a b}\right) \leq K_{0} \text {. }
$$

CASE 2: the lower boundary of $\Gamma_{j+1, k}$ is contained in the union of the upper boundaries of the large trapezoid $\widetilde{\Gamma}_{j}$ and of the small trapezoid $\Gamma_{j, N(j)}$.

In this case, using Lemma 7.3 and then Lemma 6.1 we obtain

$$
\begin{aligned}
& \text { [total strength of waves on the lower boundary of } \left.\Gamma_{j+1, k}\right] \\
& \qquad \leq 2\left(b+W_{j}\left(t_{j+1}\right)+K_{a b}\right) \leq 2\left(b+\widetilde{K}_{0}+K_{a b}\right) \leq K_{0} .
\end{aligned}
$$

Together, (8.15) and (8.16) yield (i), with $j$ replaced by $j+1$.

By induction on $j$, both of our claims are thus proved. Since the domain $\Omega_{\ell}$ can be covered with finitely many trapezoids $\widetilde{\Gamma}_{j}$ or $\Gamma_{j k}$, we conclude that the total variation of the solution $U=U(t, x)$ remains uniformly bounded, restricted to the domain $\Omega_{\ell}$.

5. The proof of Theorem 6.1 is now achieved by induction on $\ell=m+1, \ldots, m+n$. The analysis of the total variation on the domains $\Omega_{m-1}, \ldots, \Omega_{0}$ is entirely similar.

Acknowledgment. The research of the first author was partially supported by NSF, with grant DMS-1411786: Hyperbolic Conservation Laws and Applications. The research of the second author was partially supported by NSF with grant DMS-1715012.

\section{References}

[1] D. Amadori and W. Shen, Global existence of large BV solutions in a model of granular flow. Comm. Partial Differential Equations 34 (2009), 1003-1040.

[2] P. Baiti and H. K. Jenssen, Blowup in $\mathbf{L}^{\infty}$ for a class of genuinely nonlinear hyperbolic systems of conservation laws. Discrete Contin. Dynam. Systems 7 (2001), 837-853. 
[3] S. Bianchini, R. M. Colombo, and F. Monti, $L^{\infty}$ solutions for $2 \times 2$ systems of conservation laws. Riv. Math. Univ. Parma 1 (2010), 189-204.

[4] A. Bressan, Hyperbolic Systems of Conservation Laws. The One Dimensional Cauchy Problem. Oxford University Press, 2000.

[5] A. Bressan, G. Chen, Q. Zhang, Lack of BV bounds for approximate solutions to the p-system with large data, J. Differential Equations 256 (2014), 3067-3085.

[6] A. Bressan, G. Chen, Q. Zhang, and S. Zhu, No BV bounds for approximate solutions to p-system with general pressure law, J. Hyperbolic Diff. Equat. 12 (2015), 1-18.

[7] A. Bressan and R. M. Colombo, Unique solutions of $2 \times 2$ conservation laws with large data, Indiana Univ. Math. J. 44 (1995), 677-725.

[8] A. Bressan and R. M. Colombo, Decay of positive waves in nonlinear systems of conservation laws, Ann. Scuola Normale Superiore Pisa IV - 26 (1998), 133-160.

[9] A. Bressan and P. LeFloch, Structural stability and regularity of entropy solutions to hyperbolic systems of conservation laws, Indiana Univ. Math. J. 48 (1999), 43-84.

[10] A. Bressan, T. P. Liu and T. Yang, $L^{1}$ stability estimates for $n \times n$ conservation laws, Arch. Rational Mech. Anal. 149 (1999), 1-22.

[11] A. Bressan and T. Yang, A sharp decay estimate for positive nonlinear waves, SIAM Jour. Math. Anal. 36 (2004), 659-677.

[12] T. Chang and L. Hsiao, The Riemann problem and interaction of waves in gas dynamics, Longman Scientific \& Technical, Harlow, 1989.

[13] G. Chen, Optimal time-dependent lower bound on density for classical solutions of 1-D compressible Euler equations, Indiana Univ. Math. J. 66 (2017), 725-740.

[14] C. Dafermos, Generalized characteristics and the structure of solutions of hyperbolic conservation laws, Indiana Univ. Math. J. 26 (1977), 1097-1119.

[15] R. DiPerna, Singularities of solutions of nonlinear hyperbolic systems of conservation laws, Arch. Rational Mech. Anal. 60 (1975), 75-100.

[16] G. Chen and H. K. Jenssen, No TVD fields for 1-d isentropic gas flow, Comm. Partial Differential Equations, 38 (2013), 629-657.

[17] C. Cheverry, Systèmes de lois de conservation et stabilité BV. Mem. Soc. Math. France 75 (1998).

[18] R. Courant and K. O. Friedrichs, Supersonic flow and shock waves, Wiley-Interscience, New York, 1948.

[19] J. Glimm, Solutions in the large for nonlinear hyperbolic systems of equations, Comm. Pure Appl. Math. 18 (1965), 697-715.

[20] J. Glimm and P. Lax, Decay of solutions of systems of nonlinear hyperbolic conservation laws, Amer. Math. Soc. Memoir 101 (1970). 
[21] D. Hoff, Invariant regions for systems of conservation laws. Trans. Amer. Math. Soc. 289 (1985), 591-610.

[22] H. Holden and N. H. Risebro, Front Tracking for Hyperbolic Conservation Laws. SpringerVerlag, New York, 2002.

[23] H. K. Jenssen, Blowup for systems of conservation laws, SIAM J. Math. Anal. 31 (2000), 894-908.

[24] M. Lewicka, Well-posedness for hyperbolic systems of conservation laws with large BV data. Arch. Rational Mech. Anal. 173 (2004), 415-445.

[25] T. P. Liu, Linear and nonlinear large-time behavior of solutions of general systems of hyperbolic conservation laws. Comm. Pure Appl. Math. 30 (1977), 767-796.

[26] C. Moler and J. Smoller, Elementary interactions in quasi-linear hyperbolic systems, Arch. Rational Mech. Anal. 37 (1970), 309-322.

[27] T. Nishida, Global solution for an initial boundary value problem of a quasilinear hyperbolic system. Proc. Japan Acad. 44 (1968), 642-646.

[28] T. Nishida and J. Smoller, Solutions in the large for some nonlinear hyperbolic conservation laws. Comm. Pure Appl. Math. 26 (1973), 183-200.

[29] J. Smoller, Shock waves and reaction-diffusion equations, Second edition. Springer-Verlag, New York, 1994.

[30] B. Temple and R. Young. The large time stability of sound waves. Comm. Math. Phys. 179 (1996), 417-466.

[31] R. Young, Sup-norm stability for Glimm's scheme. Comm. Pure Appl. Math. 46 (1993), 903-948. 